\title{
Polymorph selection by continuous crystallization in the presence of wet milling
}

DOI:

10.1021/acs.cgd.8b01894

\section{Document Version}

Accepted author manuscript

Link to publication record in Manchester Research Explorer

\section{Citation for published version (APA):}

Li, Y., O'shea, S., Yin, Q., \& Vetter, T. (2019). Polymorph selection by continuous crystallization in the presence of wet milling. Crystal Growth \& Design. https://doi.org/10.1021/acs.cgd.8b01894

\section{Published in:}

Crystal Growth \& Design

\section{Citing this paper}

Please note that where the full-text provided on Manchester Research Explorer is the Author Accepted Manuscript or Proof version this may differ from the final Published version. If citing, it is advised that you check and use the publisher's definitive version.

\section{General rights}

Copyright and moral rights for the publications made accessible in the Research Explorer are retained by the authors and/or other copyright owners and it is a condition of accessing publications that users recognise and abide by the legal requirements associated with these rights.

\section{Takedown policy}

If you believe that this document breaches copyright please refer to the University of Manchester's Takedown Procedures [http://man.ac.uk/04Y6Bo] or contact uml.scholarlycommunications@manchester.ac.uk providing relevant details, so we can investigate your claim.

\section{OPEN ACCESS}




\title{
Polymorph selection by continuous crystallization in the presence of wet milling
}

\author{
Yang $\mathrm{Li}^{\dagger}{ }^{\dagger}$ Simon O’Shea, ${ }^{\ddagger}$ Qiuxiang Yin, ${ }^{\dagger}$ and Thomas Vetter ${ }^{*, \ddagger}$ \\ $\dagger$ School of Chemical Engineering and Technology, Tianjin University, Tianjin 300072, \\ People's Republic of China \\ $\ddagger$ School of Chemical Engineering and Analytical Science, The University of Manchester, \\ M13 9PL Manchester, United Kingdom \\ E-mail: thomas.vetter@manchester.ac.uk \\ Phone: +44161306 4370
}

\begin{abstract}
The polymorph selection in a continuous crystallization process combined with wet milling is investigated. To this end, a dimensionless population balance equation model accounting for secondary nucleation, crystal growth and breakage is formulated and solved numerically. We show that a surprisingly small number of dimensionless parameter groups (combinations of kinetic parameters and operating conditions) is decisive to control the polymorphic outcome. Specifically, we show how the operating region where the stable polymorph is obtained can be enlarged by tuning the milling intensity, feed concentration, and residence time. We further rationalize the dependence of the mean size of the particles obtained, the fraction of solute recovered and the productivity of such a process on the dimensionless variables. We showcase this for the model system L-glutamic acid crystallized from water and show that our analysis is in agreement with previously reported experimental studies. Summarizing, the analysis approach introduced here can be used to identify operating spaces for single stage
\end{abstract}


continuous crystallization processes where the right polymorph is reliably obtained and where size, solute recovery and productivity are guaranteed to desired levels.

\section{Introduction}

Polymorphism is the ability of a given compound to crystallize in at least two different crystal structures in which the crystal's building blocks, e.g., molecules, can adopt different packing arrangements and/or conformations ${ }^{1-3}$. Since polymorphs are structurally different, their stability (and therefore their solubility), as well as a multitude of other properties can vary substantially. For example, differences in hardness ${ }^{4}$, crystal shape $^{5}$, electrical and optical properties $^{6}$, dissolution and bioavailability behavior ${ }^{7,8}$, have been reported. In the case of differences in crystal shape, surface properties, and hardness, downstream processes, such as filtration, drying and tabletting, are potentially impacted by polymorphism ${ }^{5,9-11}$. Due to its effect on bioavailability and dissolution behavior, polymorphism occurring in the context of pharmaceuticals can also affect the efficacy, market competitiveness and ultimately a product's safety $^{12,13}$. On this account the design of controlled processes that ensure the consistent production of a desired polymorph with best performance properties is an important goal in the manufacturing of crystalline solids ${ }^{14-16}$.

Consequently, numerous accounts in the literature have considered the influence of different operating conditions on polymorph selection in batch processes, such as solvent selection ${ }^{17}$, supersaturation $^{18}$, temperature ${ }^{19}$, seed crystals $^{20}$, templating ${ }^{21}$, and additives $^{22}$. Kinetic and thermodynamic insights gained from such studies have been amalgamated into efficient strategies that control the polymorphic outcome of batch crystallization processes, where, most often, the stable polymorph was targeted ${ }^{23-27}$.

For polymorph selection in continuous crystallization processes, Lai et al. ${ }^{28,29}$ reported the possibility to selectively produce the targeted polymorph in a mixed suspension, mixed product removal crystallizer (MSMPRC) via stable steady-state operation for L-glutamic 
acid (LGA) and p-aminobenzoic acid polymorphs, where they reported operating conditions that reliably yield either the stable or metastable polymorph. Similarly, Agnew et al. ${ }^{30}$ successfully produced the metastable polymorph (form II) of paracetamol with high polymorph selectivity in two different continuous crystallization platforms. Motivated by the observations of Lai et al. ${ }^{28,29}$, Farmer et al. ${ }^{31}$ elucidated these results through steady state analysis. That work has shown that the polymorphic outcome in an MSMPRC process that is dominated by crystal growth and nucleation can be accurately predicted once two dimensionless stability groups - modified Damköhler numbers - are known. In essence, these dimensionless groups represent ratios of the characteristic time for nucleation and growth, the process time (residence time of the crystallizer) and the feed concentration. Their results are therefore valid for any polymorphic crystallization process conducted in an MSMPRC, as long as nucleation and growth are the dominating kinetic phenomena occurring in the process. Subsequently, the same authors have extended their analysis to cases where agglomeration occurs $^{32}$.

While agglomeration is tunable only to a certain degree in a crystallizer (by changing the hydrodynamics, supersaturation, solvent and temperature), the reverse process, i.e., crystal breakage can be induced in a targeted manner and with widely different degrees of intensity. It therefore represents an attractive "handle" to tune a process towards a desired outcome. Wet milling has been used successfully for a variety of purposes in continuous crystallization processes, such as the in situ generation of seeds through attrition/secondary nucleation ${ }^{33-35}$ and for reducing the mean size of crystals ${ }^{36,37}$. Since crystal breakage affects the number, size and therefore the surface area of crystals, it is not too surprising that an effect should be visible on the steady state behavior of a continuous crystallizer. Processes manufacturing polymorphs are no exception to this. Surprisingly, the effect of crystal breakage on the polymorphic outcome of a continuous crystallization process seems to have received limited attention so far. In this context, Köllges and Vetter ${ }^{38}$ operated a continuous crystallization process with wet milling (equivalent to the one depicted in Figure 1) and have shown that 
the use of wet milling allows to achieve the manufacture of the stable $\beta$ polymorph of LGA at operating conditions (feed concentration, crystallizer temperature and residence time) that would lead to the metastable $\alpha$ polymorph without milling. It was also shown in that paper that the use of milling increases the productivity of the overall process considerably.

While the study by Köllges and Vetter ${ }^{38}$ provided a case study on a specific substance, an in-depth study into how this phenomenon depends on the kinetics of the major mechanisms occurring in the process (nucleation, crystal growth and breakage) is still absent from the literature. To explore this, we present in Section 2 a dimensionless population balance equation and explore how the polymorphic outcome of the continuous process depicted in Figure 1 depends on key parameter groups that are amalgamations of kinetic constants, as well as operating parameters. Apart from the previously mentioned modified Damköhler numbers, we identified a surprisingly small number of additional dimensionless groups that describe the breakage intensity. The resulting model therefore allows predictions about the polymorphic form obtained in the presence of milling. In Section 3, we show how the operating space in which a desired polymorph can be reliably manufactured, changes in dependence of these dimensionless groups. Furthermore, we explore the influence of these parameters on additional product and process characteristics, such as the mean size of the crystals, as well as product recovery and process productivity. Conclusions are presented in the final section of this article.

\section{Population balance model for continuous process in} the presence of wet milling

In order to model the evolution of the particle size distribution of different polymorphs occurring in the process depicted in Figure 1 the population balance equation (PBE) framework $^{39,40}$ is used. The PBEs for both polymorphs are coupled to a solution phase mass balance describing the evolution of the liquid phase composition over time. Treating the 


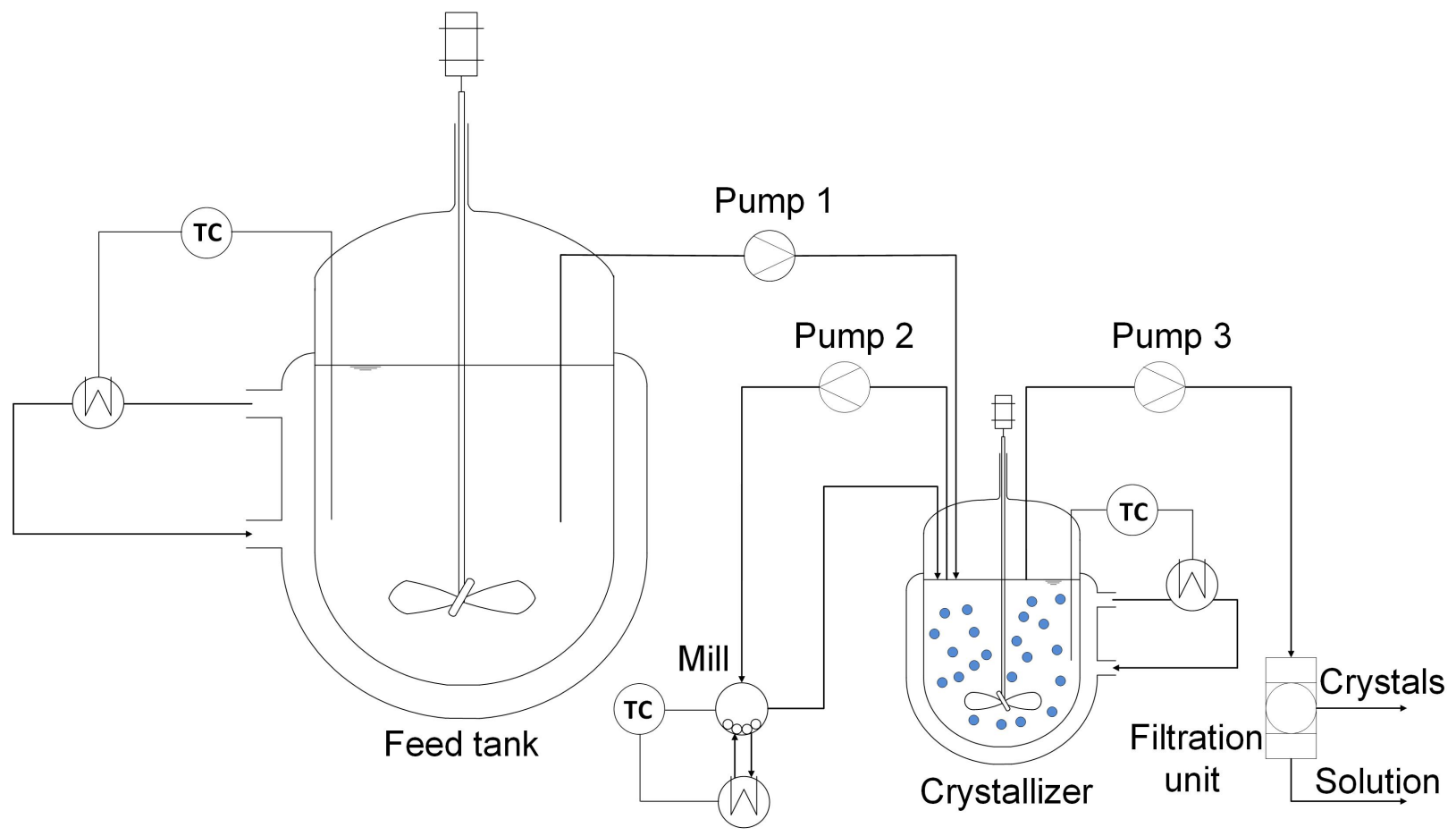

Figure 1: Process flow sheet of a continuous crystallizer with wet milling.

combination of crystallizer and mill as one well-mixed processing unit (which we will, for simplicity still refer to as MSMPRC in the remainder of this article) ${ }^{33,34,41}$, the population balance equation model for polymorph $i$ can be written as:

$$
\frac{\partial f_{i}(L, t)}{\partial t}=-\frac{f_{i}(L, t)}{\tau}-G_{i} \frac{\partial f_{i}(L, t)}{\partial L}+\int_{L}^{\infty} K_{i}(\lambda) d_{i}(\lambda, L) f_{i}(\lambda, t) \mathrm{d} \lambda-K_{i}(L) f_{i}(L, t)
$$

where $f_{i}(L, t)$ is the number density distribution for polymorph $i$ in the MSMPRC, $L$ is the characteristic size of the crystals, $t$ is time, $\tau$ is the residence time of the MSMPRC, $G_{i}$ is the size-independent growth rate, $K_{i}$ is the breakage rate of the crystals, and $d_{i}$ represents the distribution of fragments resulting from breakage ${ }^{33,41,42}$. The PBE is subject to initial 
and boundary conditions as follows:

$$
\begin{aligned}
& f_{i}(L=0, t)=\frac{J_{i}}{G_{i}} \text { for } c>c_{\mathrm{sat}, i} \\
& f_{i}(L=\infty, t)=0 \\
& f_{i}(L, t=0)=f_{\text {seed }, i}(L)
\end{aligned}
$$

where $J_{i}$ is the nucleation rate, $c$ is the solute concentration in the liquid phase, $c_{\mathrm{sat}, i}$ is the solubility of polymorph $i$ at the conditions in the MSMPRC and $f_{\text {seed, } i}(L)$ is the particle size distribution of the seed crystals. The PBE formulated in this way therefore accounts for crystal growth, nucleation, transport of particles out of the crystallizer through flow, as well as crystal breakage (induced by the mill and any other cause of breakage). Note that the boundary condition at $L=0$ only applies when the current solution phase concentration in the MSMRPC is above the solubility of polymorph $i$ (i.e., the solution is supersaturated with respect to that polymorph ${ }^{40,43}$. When this is not the case $G_{i}$ becomes a dissolution rate $\left(G_{i} \leq 0\right)$ and particles crossing $L=0$ cease to exist. In such a case, a correct description of the dynamics of the process would require the implementation of a dissolution rate for that polymorph. However, in the present work we are exclusively interested in the steady state behavior of the single stage process depicted in Figure 1. At steady state concentrations at or below the solubility of polymorph $i$, the particle size distribution of this polymorph is zero, i.e., no particles of this polymorph are present in the crystallizer. For numerical convenience, we have thus not modeled the dissolution rate of the undersaturated polymorph (we set $\left.G_{i}=0\right)$ and merely let it wash out of the crystallizer through the flow term. While this does affect the time the system requires to reach steady state, it neither affects the value of the steady state nor its stability (as others have realized and argued for already $\left.{ }^{28,29,31}\right)^{\mathrm{a}}$. The benefit of this simplified treatment is that numerical solutions of the PBE can be attained quicker. To track the change of solution concentration over time, the mass balance for the

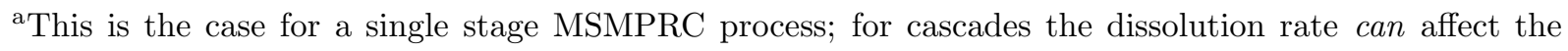
steady states obtained. 
liquid phase is written as:

$$
\frac{\mathrm{d} c(t)}{\mathrm{d} t}=\frac{c_{0}-c(t)}{\tau}-3 \sum_{i} k_{v, i} \rho_{i} G_{i} \int_{0}^{\infty} L^{2} f_{i}(L, t) \mathrm{d} L
$$

where $c_{0}$ is the feed concentration, $k_{v, i}$ indicates the volumetric shape factor, which is a dimensionless constant relating particle volume to the cube of its size ${ }^{39}$ and $\rho_{i}$ is the crystal density of polymorph $i$. In the context of this work, we are considering a system with two polymorphs, denoted $\alpha$ and $\beta$, where the $\beta$ polymorph is the stable one at all conditions. The constitutive equations used to describe the rate laws occurring in Eqs. (1) and (2) are summarized in Table 1. In summary, we are using power laws to model the crystal growth Table 1: Constitutive equations used in the population balance equation model ${ }^{\mathrm{a}, \mathrm{b}}$

\begin{tabular}{ll}
\hline mechanism & equation \\
\hline nucleation & $J_{i}=k_{n, i}\left(\frac{c}{c_{\mathrm{sat}, i}}-1\right)^{n_{i}} \mu_{2, i}$ \\
crystal growth & $G_{i}=k_{g, i}\left(\frac{c}{c_{\mathrm{sat}, i}}-1\right)^{g_{i}}$ \\
breakage rate & $K_{i}(L)=k_{\mathrm{b}, i}\left(\frac{L}{L_{0, i}}\right)^{b_{i}}$ \\
daughter distribution & $d_{i}(\lambda, L)=3 L^{2}\left(2 q_{i}+1\right)\left(\frac{2}{\lambda^{3}}\right)^{2 q_{i}+1}\left(L^{3}-\frac{\lambda^{3}}{2}\right)^{2 q_{i}}$ \\
\hline
\end{tabular}

${ }^{\mathrm{a}} \mu_{2, i}$ is the second moment of the particle size distribution of polymorph $i$, defined as: $\mu_{2, i}=\int_{0}^{\infty} L^{2} f_{i} \mathrm{~d} L$

${ }^{\mathrm{b}} L_{0, i}$ is a scaling factor which is defined as: $L_{0, i}=k_{g, i} \tau\left(\frac{c_{0}-c_{\mathrm{sat}, \beta}}{c_{\mathrm{sat}, i}}\right)^{g_{i}}$ (see below for the motivation behind this scaling factor).

rates of the polymorphs and we are assuming that nucleation proceeds through secondary nucleation exclusively. The breakage rate is assumed to scale with the size of the crystals and the fragment size distribution assumes that two fragments are formed in each breakage event (i.e., $\left.\int_{0}^{\lambda} d(\lambda, L) \mathrm{d} L=2\right)$. The daughter distribution can be tuned to represent attrition (formation of a large and small fragment, high $q_{i}$ values) and fragmentation (formation of fragments of similar size, low $q_{i}$ values).

For the purpose of reducing the number of parameters in the model, we now express the 
146

model in dimensionless form. In order to enable a comparison with the earlier work of Farmer et al. ${ }^{31}$ we closely follow their selection of scaling constants. Hence, the following variables representing dimensionless concentration $(y)$ and dimensionless time $(\xi)$ are introduced:

$$
\begin{aligned}
& y=\frac{c-c_{\mathrm{sat}, \beta}}{c_{0}-c_{\mathrm{sat}, \beta}} \in[0,1] \\
& \xi=\frac{t}{\tau}
\end{aligned}
$$

Since Farmer et al. ${ }^{31}$ considered only nucleation and crystal growth, they were able to obtain solutions to their model by formulating dimensionless moment equations. However, since breakage is considered in this work, we require solutions for the full particle size distributions occurring in the MSMPRC (at least with the constitutive equations we have chosen). We thus formulate dimensionless characteristic sizes $\left(x_{i}\right)$ and dimensionless particle size distributions $\left(h_{i}\right)$ as follows:

$$
\begin{aligned}
x_{i} & =\frac{L}{k_{g, i} \tau\left(\frac{c_{0}-c_{\mathrm{sat}, \beta}}{c_{\mathrm{sat}, i}}\right)^{g_{i}}} \\
h_{i}\left(x_{i}, \xi\right) & =\left[3 k_{v, i} k_{g, i}^{4} \tau^{4}\left(\frac{c_{0}-c_{\mathrm{sat}, \beta}}{c_{\mathrm{sat}, i}}\right)^{4 g_{i}}\left(\frac{\rho_{i}}{c_{0}-c_{\mathrm{sat}, \beta}}\right)\right] f_{i}(L, t)
\end{aligned}
$$

Inserting these quantities into the PBE of each polymorph (Eq. (1)) and into the mass balance (Eq. (3)) leads to their dimensionless equivalents:

$$
\begin{aligned}
\frac{\partial h_{\alpha}\left(x_{\alpha}, \xi\right)}{\partial \xi} & =-h_{\alpha}\left(x_{\alpha}, \xi\right)-(y+\gamma)^{g_{\alpha}} \frac{\partial h_{\alpha}\left(x_{\alpha}, \xi\right)}{\partial x_{\alpha}} \\
& +k_{\mathrm{b}, \alpha} \tau\left[\int_{x_{\alpha}}^{\infty} \epsilon_{\alpha}^{b_{\alpha}} d_{\alpha}\left(\epsilon_{\alpha}, x_{\alpha}\right) h_{\alpha}\left(\epsilon_{\alpha}, \xi\right) \mathrm{d} \epsilon_{\alpha}-x_{\alpha}^{b_{\alpha}} h_{\alpha}\left(x_{\alpha}, \xi\right)\right]
\end{aligned}
$$

$$
\begin{aligned}
\frac{\partial h_{\beta}\left(x_{\beta}, \xi\right)}{\partial \xi} & =-h_{\beta}\left(x_{\beta}, \xi\right)-y^{g_{\beta}} \frac{\partial h_{\beta}\left(x_{\beta}, \xi\right)}{\partial x_{\beta}} \\
& +k_{\mathrm{b}, \beta} \tau\left[\int_{x_{\beta}}^{\infty} \epsilon_{\beta}^{b_{\beta}} d_{\beta}\left(\epsilon_{\beta}, x_{\beta}\right) h_{\beta}\left(\epsilon_{\beta}, \xi\right) \mathrm{d} \epsilon_{\beta}-x_{\beta}^{b_{\beta}} h_{\beta}\left(x_{\beta}, \xi\right)\right]
\end{aligned}
$$




$$
\frac{\mathrm{d} y}{\mathrm{~d} \xi}=1-y-(y+\gamma)^{g_{\alpha}} \omega_{2, \alpha}-y^{g_{\beta}} \omega_{2, \beta}
$$

152

where the dimensionless group $\gamma$ is introduced that reads as:

$$
\gamma=\frac{c_{\mathrm{sat}, \beta}-c_{\mathrm{sat}, \alpha}}{c_{0}-c_{\mathrm{sat}, \beta}}<0
$$

In Eq. (8) we have used the second moment of the dimensionless particle size distribution of polymorph $i, \omega_{2, i}$, which is defined by:

$$
\omega_{2, i}=\int_{0}^{\infty} x_{i}^{2} h_{i}\left(x_{i}, \xi\right) \mathrm{d} x_{i}
$$

At conditions where the solution is supersaturated with respect to a given polymorph, Eqs. (6) and (7) are complemented with dimensionless boundary conditions:

$$
h_{\alpha}\left(x_{\alpha}=0, \xi\right)=\frac{1}{2} \operatorname{Da}_{\alpha}(y+\gamma)^{n_{\alpha}-g_{\alpha}} \omega_{2, \alpha}
$$

$$
h_{\beta}\left(x_{\beta}=0, \xi\right)=\frac{1}{2} \operatorname{Da}_{\beta} y^{n_{\beta}-g_{\beta}} \omega_{2, \beta}
$$

Here, we have used the modified Damköhler numbers, $\mathrm{Da}_{i}$, as already introduced by Farmer et al. ${ }^{31}$ :

$$
\mathrm{Da}_{i}=2 k_{n, i} k_{g, i}^{2} \tau^{3}\left(\frac{c_{0}-c_{\mathrm{sat}, \beta}}{c_{\mathrm{sat}, i}}\right)^{2 g_{i}+n_{i}}>0
$$

Using the dimensionless population balance equations and mass balance defined above, it is easy to show that - for the case without breakage, i.e., $k_{\mathrm{b}, i} \tau=0$ - the dimensionless moment equations formulated by Farmer et al. ${ }^{31}$ can be recovered.

The dynamic stability of steady states in an MSMPRC governed by the above equations is therefore in general dependent on the following dimensionless parameters or parameter groups: $\gamma, g_{i}, n_{i}, \mathrm{Da}_{i}, k_{\mathrm{b}, i} \tau, b_{i}, q_{i}$ (for two polymorphs this amounts to 13 parameters). This is obviously still a large number of parameters, but represents a reduction from the non- 
dimensionless model (21 parameters). Furthermore, by obtaining numerical solutions to the above equations, we will show that some of the dimensionless parameter (groups) are more important than others. In order to obtain the numerical solutions, we have implemented a numerical procedure based on a finite volume technique following the works by Qamar et al. ${ }^{44,45}$ and Kumar and Kumar ${ }^{46}$. The derivation of this procedure and further numerical details are given in the supplementary information.

\section{Results and discussion}

\subsection{Validation of the numerical scheme for the case without break- age}

Farmer et al. ${ }^{31}$ provided analytical solutions for moment equations resulting from the above dimensionless model when considering the case without breakage $\left(k_{\mathrm{b}, i} \tau=0\right)$. They have shown that the polymorphic outcome of the process can be represented when two dimensionless parameter groups, $\Phi_{\alpha}$ and $\Phi_{\beta}$, are known. The definitions of these stability groups read as follows:

$$
\Phi_{\alpha}=\left[\operatorname{Da}_{\alpha}^{\frac{-1}{2 g_{\alpha}+n_{\alpha}}}-\gamma\right]^{-1}
$$

$$
\Phi_{\beta}=\left[\operatorname{Da}_{\beta}^{\frac{-1}{2 g_{\beta}+n_{\beta}}}\right]^{-1}
$$

Hence, the values of $\Phi_{\alpha}$ and $\Phi_{\beta}$ are amalgamations of the model parameters $\mathrm{Da}_{i}, g_{i}, n_{i}$, and $\gamma$. In order to validate the numerical solution procedure we are using in the remainder of this article, simulations have been run for different $\Phi_{\alpha}$ and $\Phi_{\beta}$ values in the absence of breakage. Each simulation was started with a mixture of $\alpha$ and $\beta$ crystals present in a solution that is supersaturated with respect to both polymorphs. Hence, the simulations are guaranteed to converge on the dynamically stable steady state, provided they are run to sufficiently high values of the dimensionless time $\xi$. The simulation results are illustrated in Figure 2 
where the color of the dot in the $\left(\Phi_{\alpha}, \Phi_{\beta}\right)$ plane indicates the polymorph obtained (yellow dot for $\beta$ polymorph, blue dot for $\alpha$ polymorph; only polymorphically pure steady states were observed for the $\Phi_{i}$ values indicated). The analytical stability boundaries obtained by Farmer et al. ${ }^{31}$ are shown as the black solid lines. Their analysis showed that pure $\beta$ polymorph is obtained at the dynamically stable steady state for $\Phi_{\beta}>\Phi_{\alpha}$ and $\Phi_{i}>1$, while the $\alpha$ polymorph is obtained for $\Phi_{\alpha}>\Phi_{\beta}$ and $\Phi_{i}>1$. In the trivial region of $\Phi_{\alpha}<1$ and $\Phi_{\beta}<1$, no crystals exist in the crystallizer at steady state because the mass deposition rate for crystallization is smaller than the mass removal rate through the outflow of particles from the crystallizer. When $\Phi_{\alpha}=\Phi_{\beta}$, mixtures of the $\alpha$ and $\beta$ were obtained. The figure therefore indicates that our numerical results are in excellent agreement with the analytical results of Farmer et al. ${ }^{31}$.

\subsection{Mean size of crystals obtained at steady state in the case without breakage}

Apart from determining which polymorph occurs at steady state in the continuous crystallization process, the value of $\Phi_{i}$ can be used to gain further information about the process steady state as well, such as the concentration at steady state and average particle size. Based on the definition of $\Phi_{i}$, the dimensionless steady state concentration, $y_{\mathrm{ss}}$, is equal to the reciprocal value of $\Phi_{i}$, where form $i$ is the steady state polymorph. Therefore, increasing $\Phi_{i}$ results in a decrease of $y_{\mathrm{ss}}$, which means a larger fraction of solute will be recovered from the solution (as discussed later). The dimensionless average size of the steady state polymorph, $\omega_{4, i} / \omega_{3, i}$, depending on which is the steady state polymorph, can then be found $a s^{b}$ :

$$
\frac{\omega_{4, \alpha}}{\omega_{3, \alpha}}=4\left(y_{\mathrm{ss}}+\gamma\right)^{g_{\alpha}}=4\left(\frac{1}{\Phi_{\alpha}}+\gamma\right)^{g_{\alpha}}
$$

$$
\frac{\omega_{4, \beta}}{\omega_{3, \beta}}=4 y_{\mathrm{ss}}^{g_{\beta}}=4\left(\frac{1}{\Phi_{\beta}}\right)^{g_{\beta}}
$$

\footnotetext{
${ }^{\mathrm{b}}$ The detailed derivation is presented in the supplementary information
} 


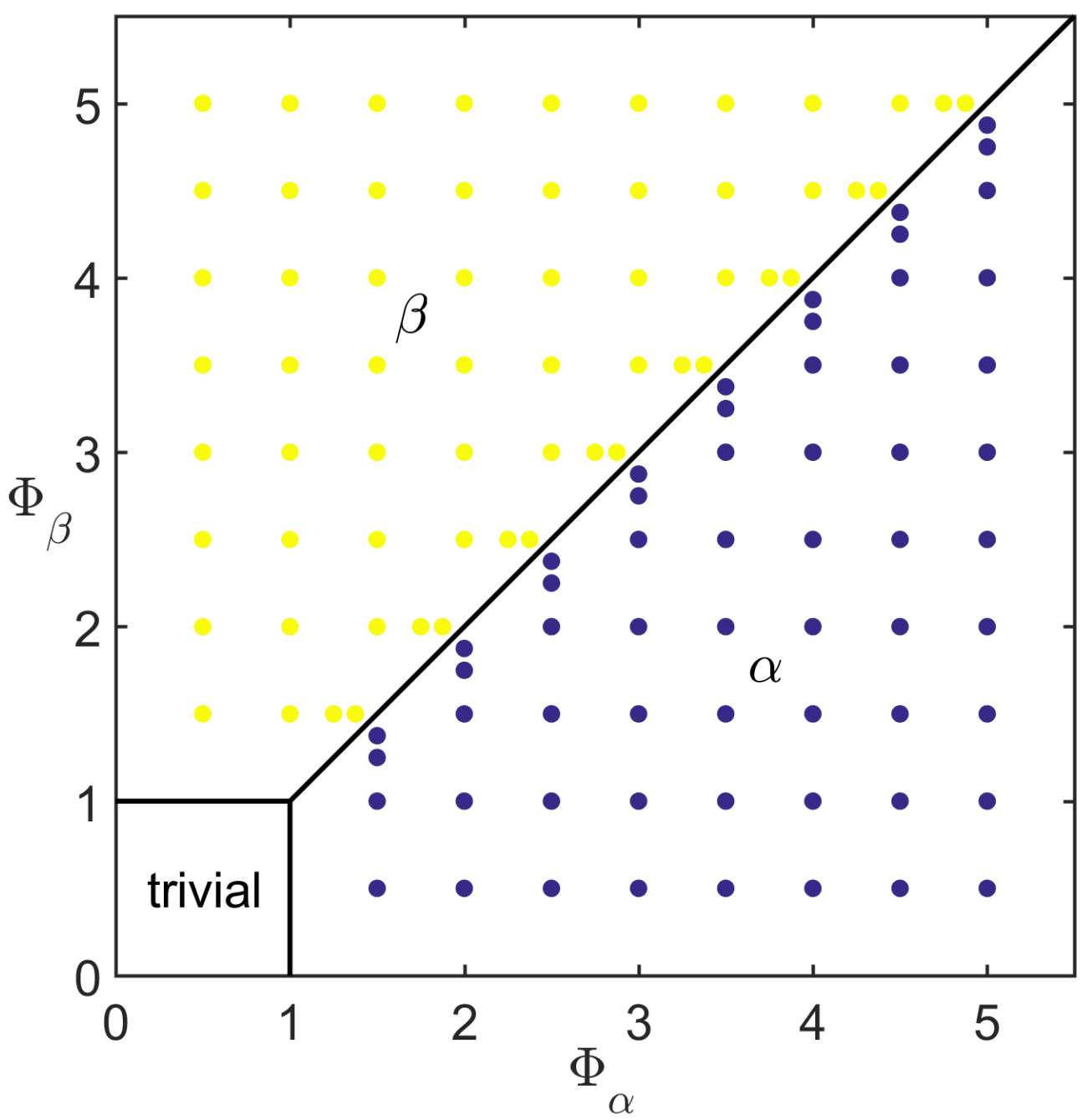

Figure 2: Stability diagram for polymorph selection without breakage. Here, each dot represents one simulation result which was run under corresponding $\Phi_{\alpha}$ and $\Phi_{\beta}$. Yellow dots indicate pure $\beta$ polymorph, while blue dots represent pure $\alpha$ polymorph. For simulations in the trivial region, there are no crystals produced at steady state.

for $\alpha$ and $\beta$, respectively.

Displaying Eqs. (16) and (17) in the $\left(\Phi_{\alpha}, \Phi_{\beta}\right)$ plane, one obtains Figure 3, where the dimensionless average size is shown using a colormap. In the region where the $\alpha$ polymorph is dynamically stable, the dimensionless average size $\omega_{4, \alpha} / \omega_{3, \alpha}$ is exclusively dependent on the parameters $\Phi_{\alpha}, g_{\alpha}$ and $\gamma$. On the other hand, for the region of the $\beta$ polymorph, only $\Phi_{\beta}$ and $g_{\beta}$ are required to determine the dimensionless average size. As demonstrated in Figure 3, the value of dimensionless average size $\omega_{4, i} / \omega_{3, i}$ is constant for constant $\Phi_{i}$ in the region 


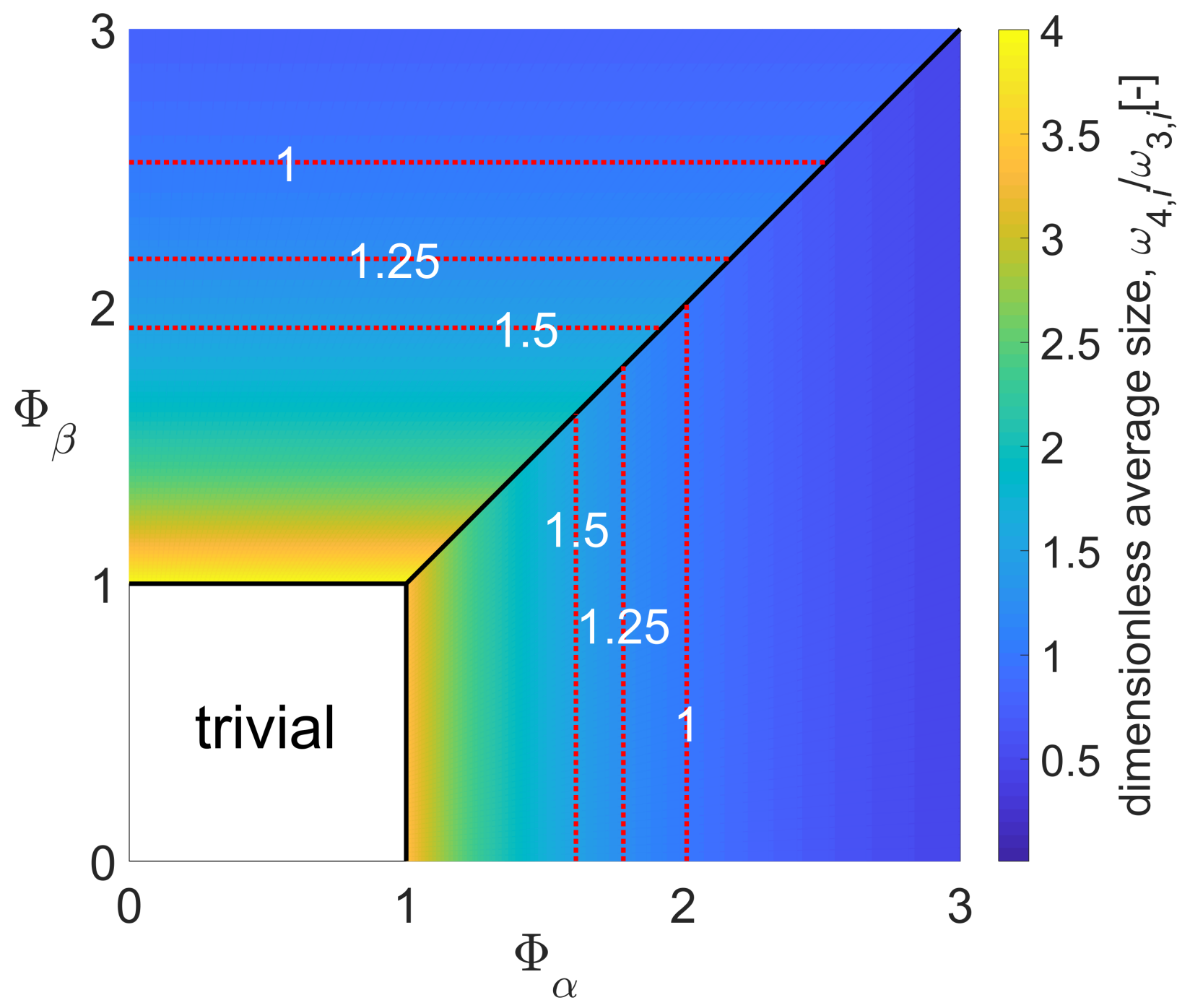

Figure 3: Dimensionless average size $\omega_{4, i} / \omega_{3, i}$ obtained in a continuous crystallizer at steady state for different $\Phi_{\alpha}$ and $\Phi_{\beta}$. The colorbar represents the value of $\omega_{4, i} / \omega_{3, i}$. The dashed isolines represent the points with the same $\omega_{4, i} / \omega_{3, i}$, as labelled. This figure shows a case with the following parameters: $\gamma=-0.1, g_{\alpha}=g_{\beta}=1.5$.

where form $i$ is dynamically stable; i.e., it does not depend on $\Phi_{k}(k \neq i)$. Furthermore, the value of the dimensionless average size $\omega_{4, i} / \omega_{3, i}$ decreases as $\Phi_{i}$ increases. Besides, for the same value of $\Phi_{\alpha}$ and $\Phi_{\beta}$, the dimensionless average size $\omega_{4, i} / \omega_{3, i}$ will always be smaller for polymorph $\alpha$ (if $g_{\alpha}=g_{\beta}$, like in the case shown in Figure 3) due to the presence of $\gamma$ in Eq. (16). $\gamma$, shown in Eq. (9), is a parameter to weigh the solubility difference between the stable and the metastable polymorph; in the way it is defined, it always yields a negative value. As a consequence, the red dashed isolines in Figure 3 illustrate that to obtain the 
same value of $\omega_{4, \alpha} / \omega_{3, \alpha}$ and $\omega_{4, \beta} / \omega_{3, \beta}$, the required value of $\Phi_{i}$ is smaller for polymorph $\alpha$ compared to polymorph $\beta$. However, it is crucial to highlight that the actual average size does not only depend on the dimensionless average size; their relationship, indeed, is the following

$$
L_{\text {mean }}=\frac{\mu_{4, i}}{\mu_{3, i}}=k_{g, i} \tau\left(\frac{c_{0}-c_{\mathrm{sat}, \beta}}{c_{\mathrm{sat}, i}}\right)^{g_{i}} \frac{\omega_{4, i}}{\omega_{3, i}}
$$

Therefore, $\tau$ and $c_{0}$ also play a major role in the value of the actual average size for polymorph $i$ in an MSMPRC at steady state at a defined temperature. The dimensionless average size and actual average size in the $\left(\Phi_{\alpha}, \Phi_{\beta}\right)$ plane will be discussed and compared below when evaluating these relationships for a specific model system.

\subsection{Results for the case with breakage}

After validating the robustness of our model for the case without breakage, we now turn to the situations with breakage. As described before, the dynamic stability of steady state in an MSMPRC is in general governed by the following dimensionless parameters or parameter groups: $\gamma, g_{i}, n_{i}, \mathrm{Da}_{i}, k_{\mathrm{b}, i} \tau, b_{i}$, and $q_{i}$. Reasonable ranges for these parameters were investigated to test the sensitivity of the stability boundary against changes in these parameters. $\gamma=-0.1,-0.2$ and -0.3 were used in the simulation process based on its expression in Eq. (9). $g_{i}$ and $n_{i}$ are exponents occurring in the power law expressions for growth rate and nucleation rate respectively. Ohara and Reid ${ }^{47}$ compiled different crystal growth models in which $g_{i}$ mainly varied from 1 to 2 . Therefore, we obtained simulation results for $g \in\{1,1.5,2\}$ in our work. Tseng and Ward ${ }^{48}$ showed an overview of different substances and parameters of power law expression for nucleation. In light of that work, the values of $n_{i}$ were selected as 1.5, 2.5 and 3.5 to cover the majority of nucleation cases. Surveying different literature sources ${ }^{36,49,50}$, one can see that $k_{\mathrm{b}, i}$, the breakage rate prefactor, can take a large range of values. Here, $k_{\mathrm{b}, i} \tau$ was combined together, as a dimensionless group, to represent different levels of breakage in an MSMPRC. The range of $k_{\mathrm{b}, i} \tau$ was selected from 0 to 7.5; 
this range of values should be well attainable in typical suspension mills for many substances. Indeed, we will show below that the mean particle sizes obtained from our model take realistic values when specifying $k_{\mathrm{b}, i} \tau$ in the mentioned range. $b_{i}$ is the exponent occurring in the power law expression for breakage rate; we tested values of $1,1.5$ and 2 for it. $q_{i}$ is the integer parameter to adjust the size of the two fragments produced by breakage, for which values of 2,5 and 10 were chosen. This covers cases from particle fragmentation, where two roughly equal-sized fragments are formed in a breakage event $\left(q_{i}=2\right)$, to attrition, where the two fragments formed by a breakage event have a large size difference $\left(q_{i}=10\right)$. The parameters for the base case used in our simulations are listed in Table 2. In the following we will in detail investigate the effect of $k_{\mathrm{b}, i} \tau$ on the polymorph selection occurring in the MSMRPC (as well as the influence of both $\Phi_{i}$ ), because it plays a major role in describing the intensity of the breakage process. The effect of the other parameters listed in Table 2 is detailed in the supplementary information.

Table 2: Parameter values for the base case ${ }^{a, b}$

\begin{tabular}{cc}
\hline parameter & value \\
\hline$g_{i}$ & 1.5 \\
$n_{i}$ & 2.5 \\
$k_{\mathrm{b}, i} \tau$ & 2.5 \\
$q_{i}$ & 2 \\
$\mathrm{~b}_{i}$ & 1 \\
$\gamma$ & -0.1 \\
\hline
\end{tabular}

a All parameters here are dimensionless values.

${ }^{\mathrm{b}} i$ is for both $\alpha$ and $\beta$ polymorph.

We start our discussion by assuming that the two polymorphs break equally, i.e., $k_{\mathrm{b}, \alpha} \tau=$ $k_{\mathrm{b}, \beta} \tau$. The stability boundaries resulting in this case are displayed in Figure 4(a). The black lines indicate the stability boundaries of the reference case without breakage. The red lines show the stability boundaries obtained at increasing values of $k_{\mathrm{b}, i} \tau$ (holding all other parameters constant). One major trend is that the stability region of the $\alpha$ polymorph shrinks for increasing values of the breakage parameter - even though the two polymorphs 
are breaking equally. That means that the operating region where the stable polymorph ( $\beta$ polymorph) can be obtained becomes larger. In addition, one can see that the low $\Phi_{i}$ area is more sensitive to this trend compared to areas of high $\Phi_{i}$. Interestingly, the area of the trivial steady state (where no particles are present at steady state; $0<\Phi_{\alpha}<1$ and $\left.0<\Phi_{\beta}<1\right)$ is strongly affected by introducing breakage as well. In order to identify the position of the boundaries of this trivial area from our numerical solutions, we have identified the combination of $\Phi_{i}$ values where the dimensionless steady state concentration, $y_{\mathrm{ss}}$, becomes larger than $0.999^{\mathrm{c}}$. From the inset in Figure 4(a), we can see that the trivial region shrinks with increasing values of the breakage parameter. Ultimately, at high enough values of $k_{\mathrm{b}, i} \tau$, the trivial steady state disappears completely from the $\left(\Phi_{\alpha}, \Phi_{\beta}\right)$ plane. Since breakage introduces an additional way to generate particles in the crystallizer (in addition to nucleation) and it increases surface area (at constant particle mass), it makes sense that lower $\Phi_{i}$ values lead to steady states with a sustainable particle population when breakage is present.

Since the two polymorphs can have different morphologies and mechanical properties, it is likely that they break with different rates. We are investigating this more general case in Figure 4(b)-(c). Investigating the extreme case where only one of the two polymorphs breaks (either $\beta$ or $\alpha$ ) yields the stability boundaries reported in Figure 4(b) and (c), respectively. When only the stable polymorph undergoes breakage (Figure 4(b)), the behavior of the stability boundary with respect to increasing $k_{\mathrm{b}, \beta} \tau$ is qualitatively similar to the case where both polymorphs break equally. However, the same value of $k_{\mathrm{b}, \beta} \tau$ shifts the boundary deeper into the $\alpha$ region. Conversely, when only the metastable $\alpha$ polymorph breaks (Figure 4(c)), the stability region of the $\alpha$ polymorph expands at low $\Phi_{i}$ values, while it remains similar to the case without breakage at high $\Phi_{i}$ values. When both polymorphs break, but at different rates, cases such as the one depicted in Figure 4(d) are obtained, where we have kept $k_{\mathrm{b}, \beta} \tau$ constant, but varied $k_{\mathrm{b}, \alpha} \tau$. Here, the stability boundary is shown to be able to cross the

${ }^{\mathrm{c}}$ In other words, this means that we have identified the position of these boundaries to within 0.001 in the $\left(\Phi_{\alpha}, \Phi_{\beta}\right)$ plane. 


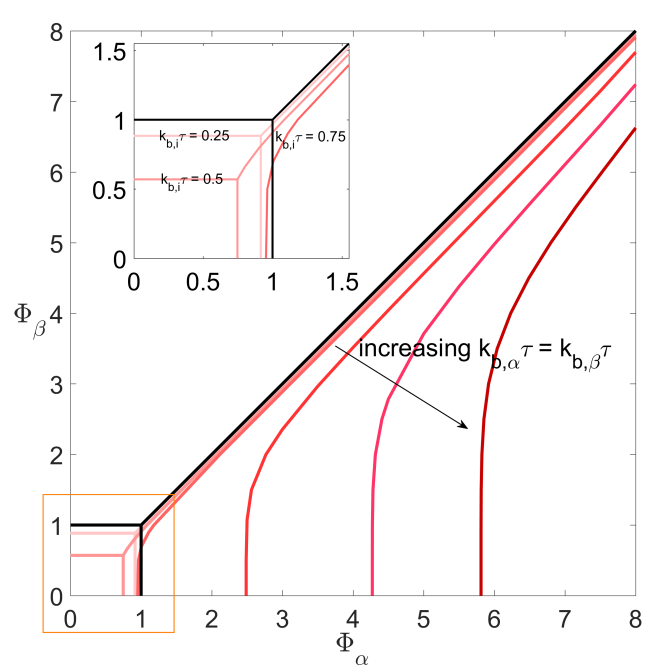

(a)

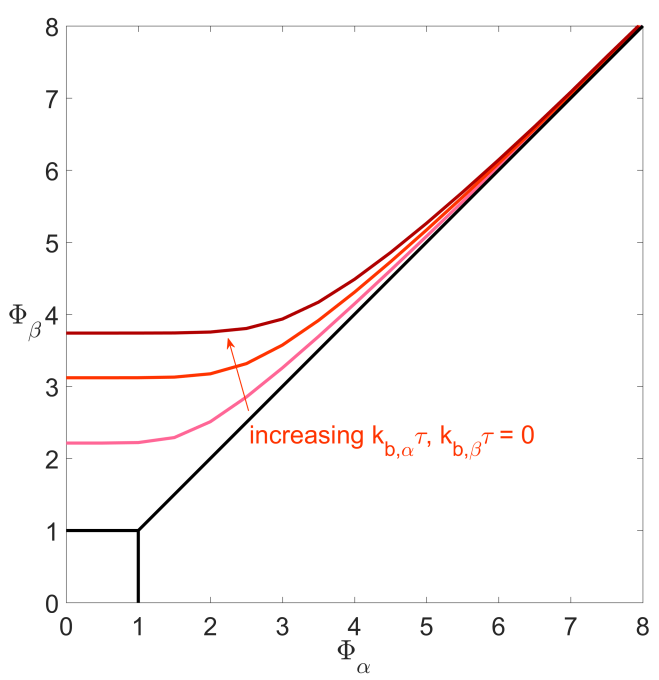

(c)

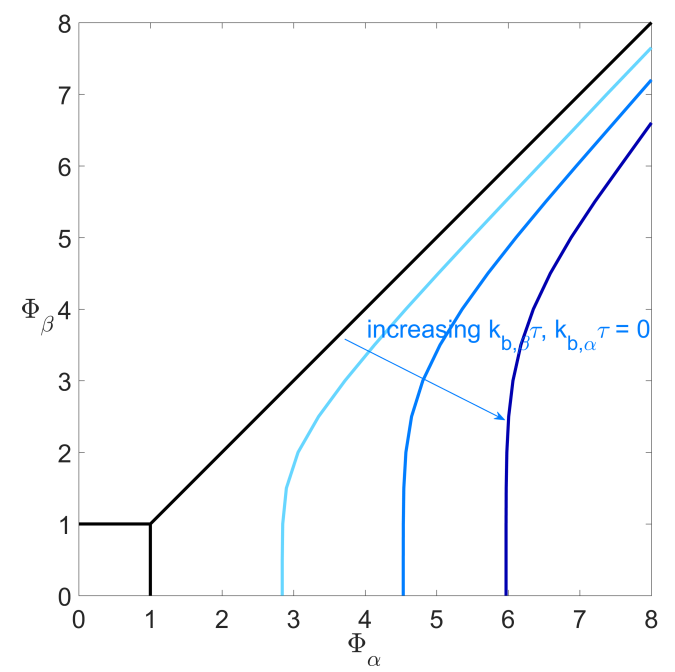

(b)

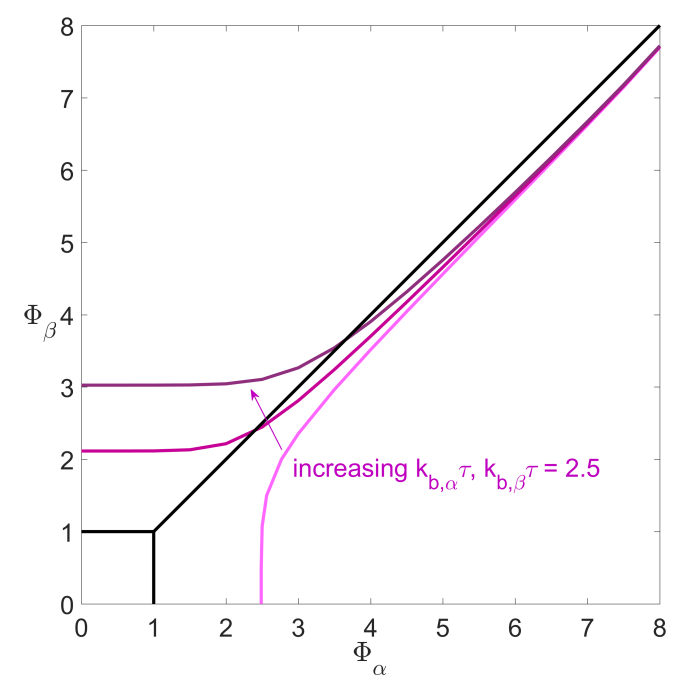

(d)

Figure 4: Maps of polymorph stability in the $\left(\Phi_{\alpha}, \Phi_{\beta}\right)$ plane for different breakage intensities. (a) Both polymorphs breaking with equal rates $\left(k_{\mathrm{b}, \alpha} \tau=k_{\mathrm{b}, \beta} \tau \in\{0.25,0.5,0.75,2.5,5,7.5\}\right)$. (b) Only the $\beta$ polymorph breaks $\left(k_{\mathrm{b}, \alpha} \tau=0, k_{\mathrm{b}, \beta} \tau \in\{2.5,5,7.5\}\right)$. (c) Only the $\alpha$ polymorph breaks $\left(k_{\mathrm{b}, \beta} \tau=0, k_{\mathrm{b}, \alpha} \tau \in\{2.5,5,7.5\}\right)$. (d) Both polymorphs break, but with different rates $\left(k_{\mathrm{b}, \beta} \tau=2.5, k_{\mathrm{b}, \alpha} \tau \in\{2.5,5,7.5\}\right)$. The black lines show the stability boundaries for the case without breakage in each subfigure. The inset in (a) shows the trivial region enlarged.

$\Phi_{\alpha}=\Phi_{\beta}$ line. Furthermore, in such cases the influence of breakage on polymorph selection at low $\Phi_{i}$ values becomes dependent on the specific combination of values used, while the behavior is again dominated by the stable polymorph at larger $\Phi_{i}$ values.

As illustrated in Figure 4(a), the trivial region, in which no crystals are present at steady state, shrinks with increasing breakage intensity (increasing $k_{\mathrm{b}, i} \tau$ ). To investigate this fur- 
ther, we will now consider the case where new crystals are solely formed through breakage, i.e., we investigate how this trivial region behaves when setting the nucleation rates of both polymorphs to zero $\left(k_{n, i}=0\right.$, which also means $\left.\mathrm{Da}_{i}=0\right)$. The results of such simulations are shown in Figure 5. There is a trivial region (where no crystals are present) in the lower left corner in both panels (yellow rectangle in (a), white rectangle in (b)). The stability boundary is shown as the black dashed line. In the left panel the colormap shows the dimensionless concentration at steady state, while in the right panel it shows the dimensionless average size. One can see that above a specific breakage intensity (above specific values of $k_{\mathrm{b}, i} \tau$ ) steady states with a sustainable population of either $\alpha$ or $\beta$ crystals are obtained ${ }^{\mathrm{d}}$. This indicates that the addition of breakage to the process can lead to acceptable process performance even for very poorly nucleating compounds. Considering the left panel displaying the dimensionless concentration at steady state in more detail, we see that the contour lines are vertical in the region where the $\alpha$ polymorph is produced and horizontal in the region where the $\beta$ polymorph is produced. This indicates again that (apart from the values on the black line) the steady states obtained are polymorphically pure. Furthermore, as expected, the results indicate that increasing breakage intensity leads to a process with higher product recovery. The panel on the right shows the dimensionless average size obtained from the processes. As expected, we see that the contour lines are vertical/horizontal in the respective regions and that the particles become smaller the higher the breakage intensity.

\subsection{Application to a model system: L-glutamic acid crystallized from water}

To illustrate the approach presented above and supplement it with a case study, we now study the cooling crystallization process of the model compound L-glutamic acid (LGA) crystallized from water as a solvent. We will first introduce the case study and illustrate

\footnotetext{
${ }^{\mathrm{d}}$ The numerical value of $k_{\mathrm{b}, i} \tau$ where this transition occurs depends on the functional forms of the breakage rate and the daughter distribution, as well as the value of $\gamma$.
} 


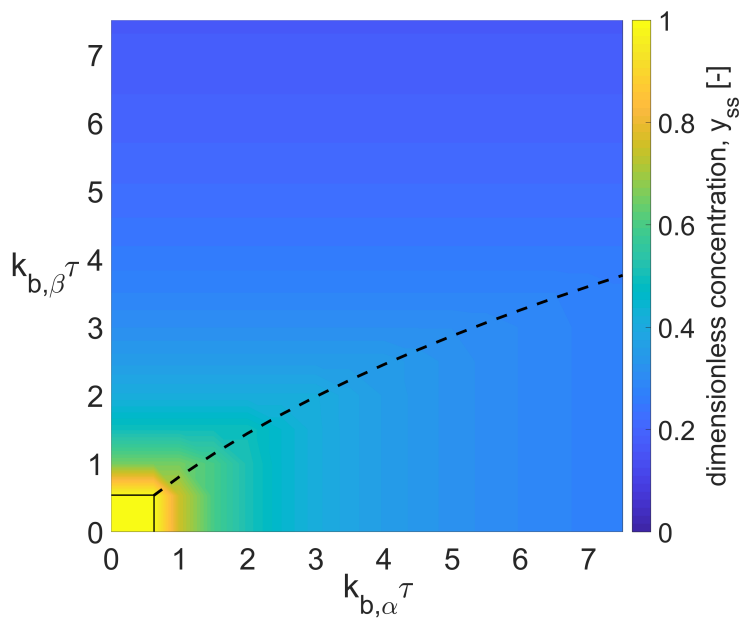

(a)

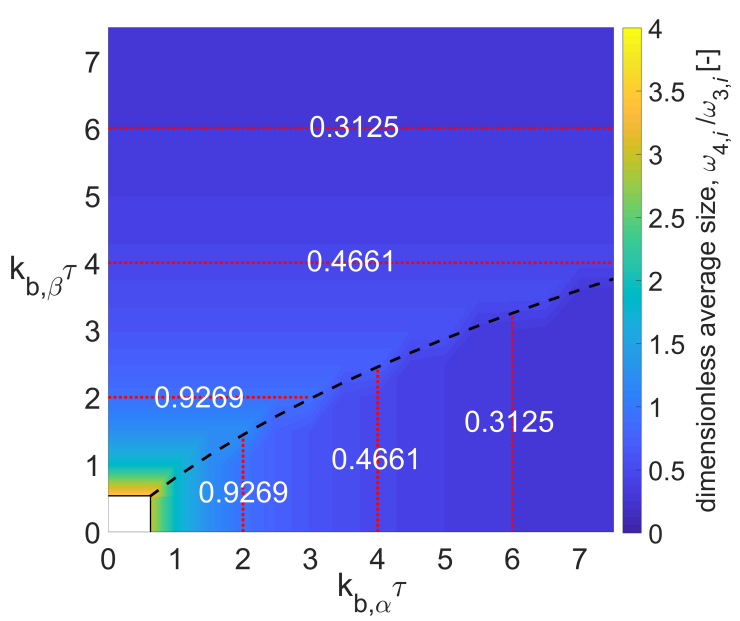

(b)

Figure 5: Maps of polymorph stability in the $\left(k_{\mathrm{b}, \alpha} \tau, k_{\mathrm{b}, \beta} \tau\right)$ plane for the case where neither polymorph is able to nucleate, i.e., new crystals are solely formed through breakage $\left(k_{\mathrm{n}, i}=0\right.$, hence $\left.\mathrm{Da}_{i}=0\right)$. The black lines indicate the stability boundaries. The yellow region in (a) and the white region in (b) indicate the trivial region where no crystals are present at steady state, which is limited by the black solid lines. The colored area below the dashed black line is the region where the $\alpha$ polymorph is obtained, whereas the $\beta$ polymorph is obtained in the colored region above the dashed black line. The colormap indicates the dimensionless steady state concentration in (a), whereas it represents the dimensionless mean size of the polymorph obtained in (b). Contour lines for specific values of the dimensionless average size are also shown with red dotted lines in (b).

the region in the $\left(\Phi_{\alpha}, \Phi_{\beta}\right)$ plane that is accessible for this system (Section 3.4.1), and then proceed to analyze in which region each polymorph can be manufactured for the case without crystal breakage (Section 3.4.2) and for the case with crystal breakage (Section 3.4.3). For the two latter sections, we also analyze the mean sizes, fraction of product recoverable and process productivity.

\subsubsection{Introduction to the case study}

LGA crystallizes in two polymorphs; the stable $\beta$ polymorph and the metastable $\alpha$ polymorph, which are monotropically related. The kinetics and solubilities of both forms have been well-reported in the literature ${ }^{28,51-58}$. Specifically, in the context of continuous crystallization, Lai et al. ${ }^{28}$, as well as Farmer et al. ${ }^{31,32}$ have presented a contiguous set of experimental and/or model data. Recently, model and experimental results including break- 
age have been presented by Köllges and Vetter ${ }^{38}$ as well. By choosing the model substance and specifying the crystallizer temperature $\left(25^{\circ} \mathrm{C}\right.$ throughout the rest of this work), the physical properties and the solubilities of both polymorphs become determined. Furthermore, the parameters appearing in the nucleation and growth rate expressions become fixed. An overview of these values is reported in Table 3. This leaves the feed concentration, the residence time in the crystallizer, as well as the breakage intensity as operating conditions that can be chosen. As laid out above, the combination of these values will determine the polymorphic outcome, the particle size distribution obtained, and in turn the productivity and product recovery of the process. In a cooling crystallization, the upper bound of the feed concentration is given by the thermal stability of the solute or the solubility of the solute at the boiling point of the solvent (whichever occurs at lower temperature). Conversely, the feed concentration is also subject to a lower bound, because a meaningful crystallization process has to be fed with a solution that is supersaturated (with respect to at least one polymorph) at the temperature the crystallizer is operated at. Hence, we have chosen the lower limit of the feed concentration, $c_{0}$, as $10 \mathrm{~kg} \mathrm{~m}^{-3}$ which is slightly higher than the solubility of the $\beta$ stable polymorph at $25^{\circ} \mathrm{C}$. The upper limit of the feed concentration was chosen as $50 \mathrm{~kg} \mathrm{~m}^{-3}$ water (roughly the solubility of the polymorph $\beta$ at $75^{\circ} \mathrm{C}^{52,57}$; note that LGA was recently shown to undergo degradation at temperatures in excess of this value ${ }^{38}$ ). The range of residence times realizable in an MSMPRC is bounded by considerations regarding the transport of suspension from the crystallizer (e.g., the flow velocity in the outlet pipe has to be chosen such that size classification does not occur, that particles get transported efficiently from the crystallizer and, of course, the particles have to fit through the pipes without clogging them). As such, the feasible range of residence times is indirectly dependent on the scale of the process. Here, we are choosing a generous (i.e., wide) range from 0.1 to $100 \mathrm{~h}$.

With the crystallization kinetics and the physical properties specified and the operating conditions bounded, the region of operating conditions for LGA can be readily displayed 
Table 3: Nucleation and growth kinetics parameters and LGA crystal properties used in this work $^{\mathrm{a}, \mathrm{b}, \mathrm{c}}$

\begin{tabular}{ccc}
\hline parameter & $\alpha$ polymorph & $\beta$ polymorph \\
\hline$k_{g, i}$ & $1.3 \times 10^{-8} \mathrm{~m} \mathrm{~s}^{-1}$ & $1.6 \times 10^{-9} \mathrm{~m} \mathrm{~s}^{-1}$ \\
$g_{i}$ & 1.31 & 1.10 \\
$k_{n, i}$ & $3.81 \times 10^{4} \mathrm{~m}^{-2} \mathrm{~s}^{-1}$ & $7.95 \times 10^{2} \mathrm{~m}^{-2} \mathrm{~s}^{-1}$ \\
$n_{i}$ & 2.62 & 2.81 \\
$k_{v, i}$ & $\pi / 6$ & 0.01 \\
$\rho_{i}$ & $1532 \mathrm{~kg} \mathrm{~m}^{-3}$ & $1569 \mathrm{~kg} \mathrm{~m}^{-3}$ \\
$c_{\mathrm{sat}, i}$ & $11.36 \mathrm{~kg} \mathrm{~m}^{-3}$ & $8.51 \mathrm{~kg} \mathrm{~m}^{-3}$ \\
\hline
\end{tabular}

a Data of $k_{g, i}, g_{i}, h_{i}$ and $k_{\mathrm{v}, i}$ were all taken from Lai et al. ${ }^{28}$, the value of nucleation rate pre-factor $k_{n, i}$ was obtained by using higher fidelity adjustment factors from Farmer et al. ${ }^{32}$.

b The density of polymorph $\alpha$ and $\beta$ were gained from Lehmann and Nunes ${ }^{59}$ and Hirokawa $^{60}$, respectively.

c The saturated solubility of polymorph $\alpha$ and $\beta$ were fitted from Manzurola and Apelblat $^{52}$, Mo et al. ${ }^{57}$.

into the $\left(\Phi_{\alpha}, \Phi_{\beta}\right)$ plane, see the green area in Figure 6. Every point in this area can be chosen by specifying the residence time and feed concentration in the crystallizer. The area is bounded by different combinations of the lower and upper bounds of the feed concentration and residence time, see the blue and red solid lines and their annotations in the figure. We have also drawn lines in the inner part of this region to indicate the movement of the steady state operating point when increasing residence time at constant feed concentration (blue dashed line) and when increasing feed concentration at constant residence time (red dashed line).

\subsubsection{Analysis of polymorph stability, product recovery and process productiv- ity for the case without breakage}

As discussed in Farmer et al. ${ }^{31}$, it is evident that the stability areas of the $\alpha$ and $\beta$ polymorph (their boundaries are given in Figure 6 as the black lines) are accessible by choosing the residence time and feed concentration, i.e., the desired polymorph can be "dialed-in" in a targeted manner. 


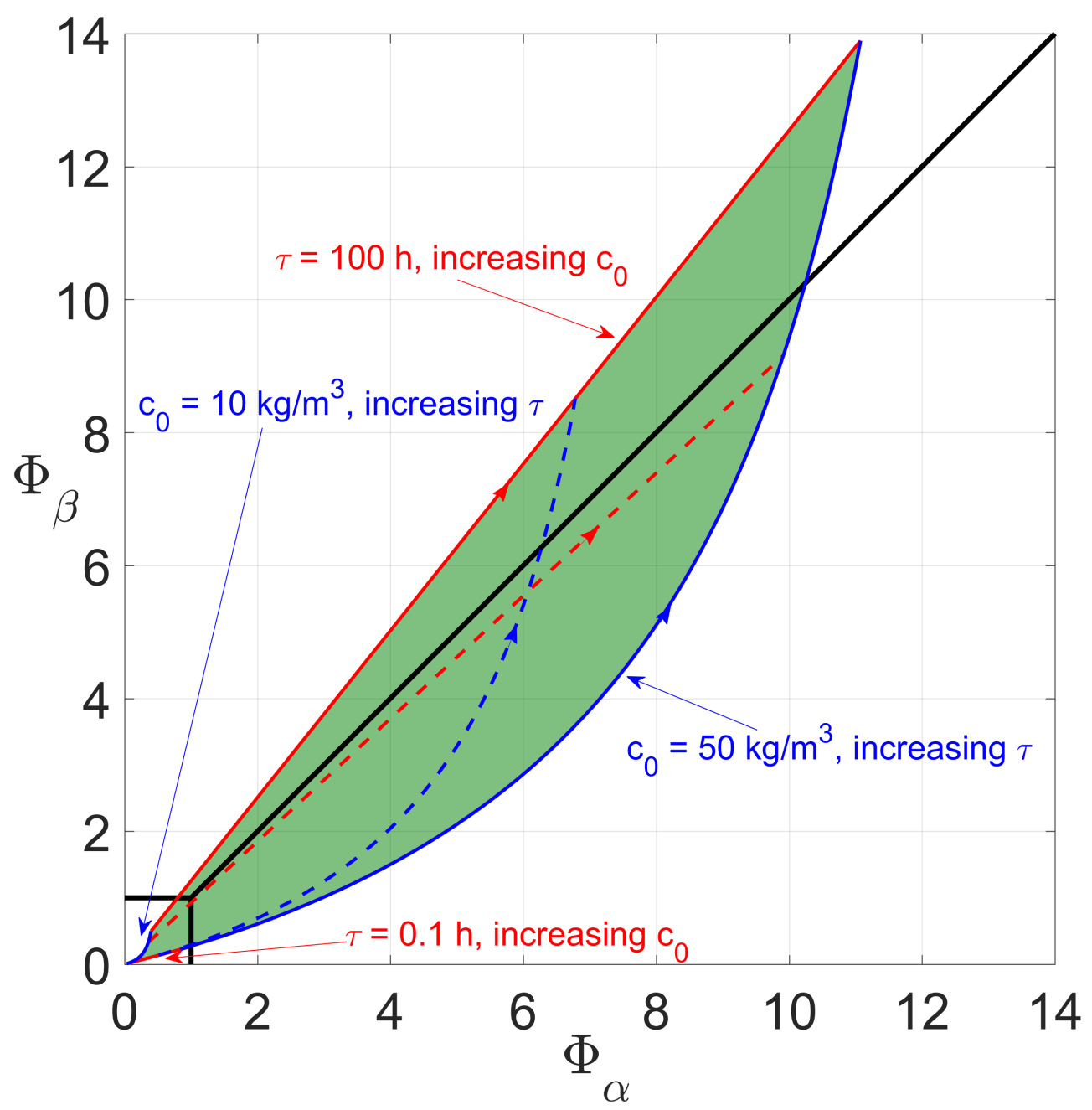

Figure 6: Feasible operating points for a single stage MSMPRC process in the $\left(\Phi_{\alpha}, \Phi_{\beta}\right)$ plane for the continuous crystallization of LGA from water at $25^{\circ} \mathrm{C}$. The green region encompassing all operating points is limited by different upper and lower bounds set for the residence time and feed concentration (see annotations). The blue dashed line indicates the movement in the $\left(\Phi_{\alpha}, \Phi_{\beta}\right)$ plane when increasing residence time at constant feed concentration; similarly, the red dashed line shows the movement with increasing feed concentration at constant residence time. The black solid lines are the boundaries of the different stability regions.

Apart from the polymorph that is obtained at steady state, further characteristics of the process and of the produced particles may, however, be important. To exemplify this, we will proceed to consider the mean size of the crystals obtained, the fractional recovery of the solute, as well as the process productivity obtained from every feasible operating point. These properties are readily calculated from the dimensionless quantities appearing in the process model. The equation for the volume-weighted mean size was already given above 
(Eq. (18)). The fraction of product recovered, $R$, can be calculated from the dimensionless concentration at steady state and the operating parameters:

$$
R=\frac{c_{0}-c_{\mathrm{ss}}}{c_{0}}=\frac{\left(1-y_{\mathrm{ss}}\right)\left(c_{0}-c_{\mathrm{sat}, \beta}\right)}{c_{0}}
$$

where $c_{\mathrm{ss}}$ is the steady state concentration in the crystallizer. Similarly, the productivity of the process (mass of crystals produced per process volume per time), $P$, can be calculated as:

$$
P=\frac{c_{0}-c_{\mathrm{ss}}}{\tau}=\frac{\left(1-y_{\mathrm{ss}}\right)\left(c_{0}-c_{\mathrm{sat}, \beta}\right)}{\tau}
$$

Focussing first on the case without breakage, we are reporting the average size and the fraction of product recovered, as well as the dimensionless average size and the dimensionless steady state concentration in Figure 7. Figure 7(a) shows the dimensionless average size $\left(\omega_{4, i} / \omega_{3, i}\right)$ for every feasible combination of $\left(\Phi_{\alpha}, \Phi_{\beta}\right)$, while Figure $7(\mathrm{~b})$ shows the average size $\left(\mu_{4, i} / \mu_{3, i}\right)$. Note that, due to the relationship detailed in Eq. (18), the dimensionless average size and the average size follow different trends when displayed in the $\left(\Phi_{\alpha}, \Phi_{\beta}\right)$ plane. One can see from these figures that manufacturing small particles of the stable $\beta$ polymorph is difficult with the average sizes that can be obtained from the process being in a relatively narrow interval of $550-750 \mu \mathrm{m}$. Furthermore, the smallest average sizes that can be obtained for the stable $\beta$ polymorph are located close to the stability boundary. Since kinetic parameters are typically only known within certain confidence intervals ${ }^{61}$ and the operating conditions are only controlled to a certain precision, the position in the $\left(\Phi_{\alpha}, \Phi_{\beta}\right)$ plane is subject to uncertainty. Aiming for (potentially desirable) small particles of the $\beta$ polymorph in the process without breakage, i.e., operating close to the stability boundary, is therefore problematic. Conversely, the average sizes attainable for the $\alpha$ polymorph span a larger range (roughly between 200 and $600 \mu \mathrm{m}$ ). However, when considering the dimensionless concentration at steady state and the fraction of product recovery derived from it (Figure 7(c) and (d)), one can see that the smaller sizes are only accessible when the product recovery is 
small (i.e., at small $\Phi_{\alpha}$ values). This means that the stream exiting the crystallizer would still be considerably supersaturated, which would potentially lead to downstream processing problems (e.g., intergrowth of the crystals during isolation on a filtration unit). At larger $\Phi_{i}$ values, reasonable product recoveries of up to $80 \%$ are feasible. Note that the calculation of the fraction of product recovered is based on the crystalline product only, i.e., not including the solute molecules remaining in the mother liquor. A further increase of the fraction of product recovered would require recycling (part of) the mother liquor ${ }^{62,63}$. By combining the information of these figures, one can conclude that reasonable rates of product recovery are linked with large average particles sizes for the case without breakage.

\subsubsection{Analysis of polymorph stability, product recovery and process productiv- ity for the case with breakage}

We will now consider the average size $\left(L_{\text {mean }}\right)$, fraction of product recovered $(R)$ and the process productivity $(P)$ for the single stage MSMPRC process carried out in the presence of different breakage intensities; the relevant figures are displayed in Figure 8 (to facilitate a comparison, we have also included the figures for the case without breakage). In this figure, we have drawn the stability boundaries as black lines (solid for the case without breakage, dashed for the case with breakage). One can see that the line separating the region where the $\alpha$ polymorph is obtained from the region where the $\beta$ polymorph shifts downwards and to the right in the $\left(\Phi_{\alpha}, \Phi_{\beta}\right)$ plane; similarly to the cases discussed before (cf. Figure 4$)$. While the boundary still falls within the feasible operating region for LGA at $k_{\mathrm{b}, i} \tau=2.5$, the boundary has been shifted outside the feasible region for the cases with higher breakage intensities. Therefore, this analysis indicates that at high breakage intensities only the $\beta$ polymorph can be obtained, whatever the feed concentration and residence time selected from the feasible operating region. This finding is in good agreement with the experimental data reported in Köllges and Vetter ${ }^{38}$, where it was shown that the residence time and feed concentration range where the $\beta$ polymorph can be obtained in a stable fashion was enlarged through the 


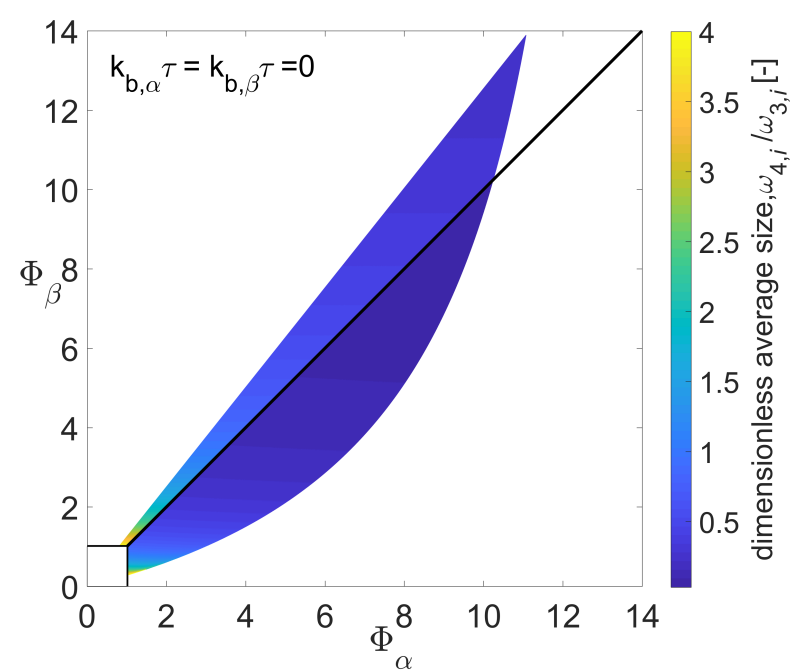

(a)

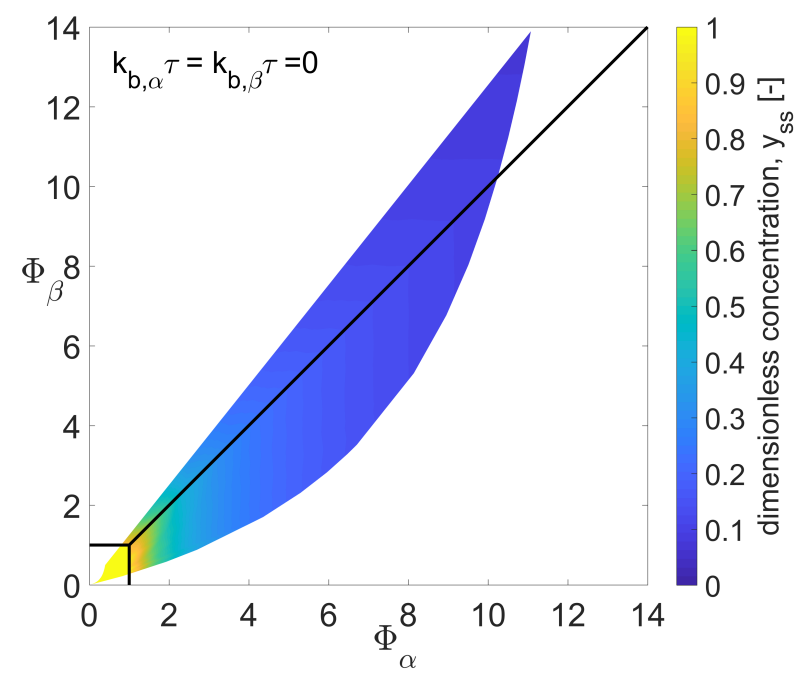

(c)

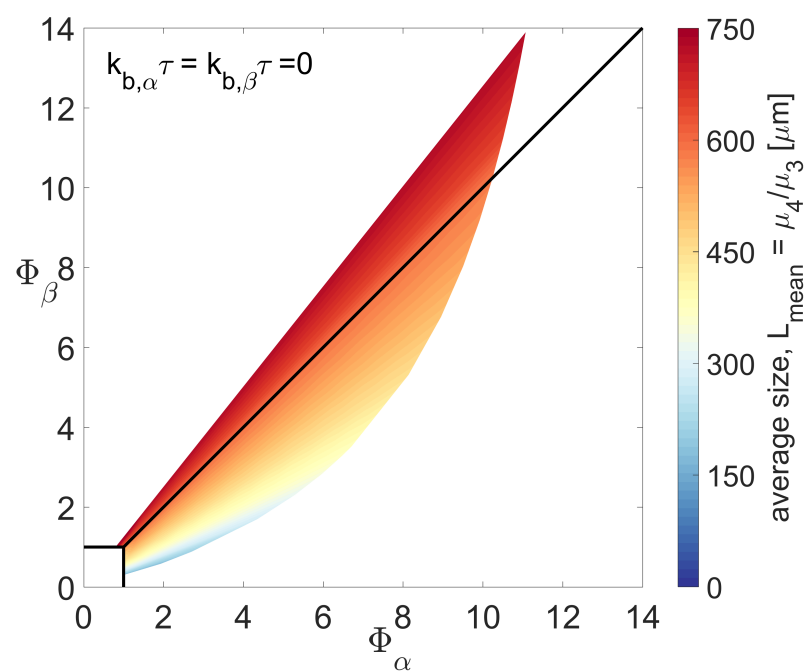

(b)

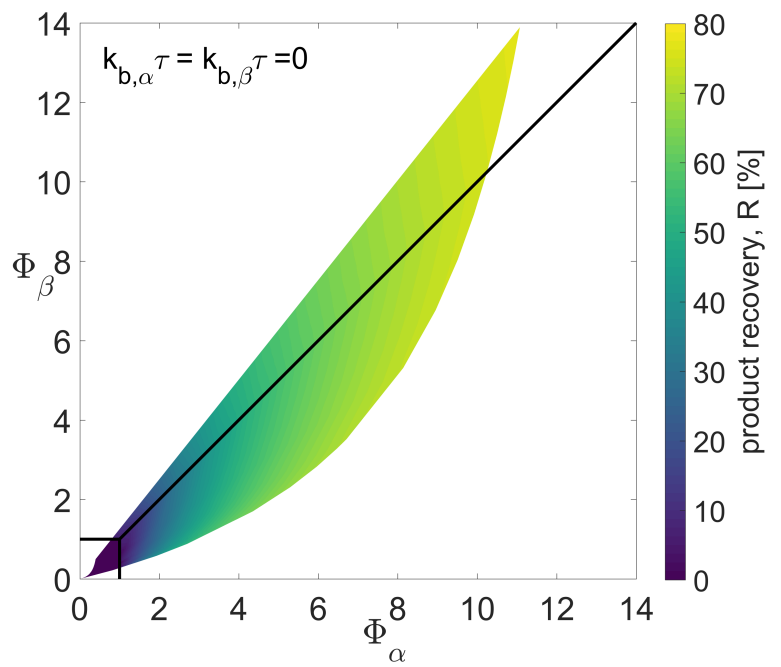

(d)

Figure 7: Different product/process characteristics for a single stage MSMPRC operated without milling depicted in the $\left(\Phi_{\alpha}, \Phi_{\beta}\right)$ plane. Only results belonging to the feasible operating region of LGA crystallized from water at $25^{\circ} \mathrm{C}$ are shown. (a) Dimensionless average size $\left(\omega_{4, i} / \omega_{3, i}\right)$, (b) average size $\left(\mu_{4, i} / \mu_{3, i}\right)$, (c) dimensionless steady state concentration, (d) product recovery. Note that no crystals are present in the region where $\Phi_{\alpha}<1$ and $\Phi_{\beta}<1$, so that the mean sizes (panels (a) and (b)) are left blank in that region. As before, the $\alpha$ polymorph is found at the dynamically stable steady state when $\Phi_{\alpha}>\Phi_{\beta}$ (and vice versa) for the remaining feasible operating points.

use of a suspension mill.

Focussing now on the mean size (left panels in Figure 8), one can, unsurprisingly, see that smaller particle sizes become accessible for the $\beta$ polymorph with increasing breakage in- 
tensity (down to a few tens of microns). Conversely, this analysis also shows that small particles of the $\alpha$ polymorph cannot be obtained in this single MSMPRC process by introducing breakage, because the stability boundary shifts out of the feasible operating region for LGA with increasing breakage intensity. That means that, if one operates at a steady state generating $\alpha$ particles and introduces breakage in order to decrease particle size, one actually induces a switch in polymorphism - one might obtain small particles in the end, but they would be of the $\beta$ polymorph! We would also like to point out that there is a discontinuity in mean size obtained when crossing the stability boundary; this is most visible in panel (d), but also to a lesser degree in panel (a), which is consistent with earlier observations on the dimensionless mean size (cf. Figure 5).

In the middle panels of Figure 8, one can see that the fraction of product recovered becomes higher in large swaths of the feasible region when increasing the breakage intensity. Though, the maximum value still lies at roughly $80 \%$ since the steady state concentration cannot fall below the solubility of the stable $\beta$ polymorph. Trying to go beyond this thermodynamic limitation by tuning the operating conditions is a futile exercise. Recovering more of the solute would require operation at a lower temperature, in a different solvent, or would require introducing a means for mother liquor recycling ${ }^{62,63}$. Considering further the productivity of the process (right panels in Figure 8; note that the colorscale is logarithmic in this case), we can see that the process operated with breakage allows obtaining reasonable productivities for either polymorph as long as the operating point is chosen correctly. For the sake of making an example, to run the process at high productivity and high fraction of solute recovery, one could operate the process with feed concentration/residence time combinations that give $\Phi_{\alpha} \approx 6$ and $\Phi_{\beta} \approx 4$ (for LGA, this corresponds to residence times of a few hours and high feed concentrations, cf. Figure 6). By choosing the breakage intensity, one can then choose the polymorph obtained (e.g., increasing breakage intensity and moving from panels (e) and (f) to (h) and (i) yields a switch in polymorph obtained; either one can be obtained at reasonable values of productivity/recovery). Summarizing the contents of Figure 8, we can 
conclude that the single MSMPRC process gains considerable flexibility when introducing breakage through a milling loop. As a result, new combinations of polymorph, mean size of particles, productivity and solvent recovery are enabled.

\section{Conclusions}

This paper presents a mathematical model for continuous crystallization processes involving wet milling and investigates the crystallization of polymorphic substances. Similar to earlier works targeting the elucidation of polymorphic behavior in continuous crystallizers (for cases of nucleation and growth only ${ }^{31}$ and for the case of nucleation, growth and agglomeration ${ }^{32}$ ), we have made the resulting population balance equations dimensionless. This led to a surprisingly small number of parameter groups (combinations of operating conditions and kinetic parameters) that control the polymorphic outcome of the process. Specifically, we have shown that introducing breakage (milling) to the process, the polymorph that breaks more readily can be obtained in a wider range of operating conditions (feed concentration, residence time) and have analyzed this behavior in detail. In contrast to the earlier works on this topic, we have also considered additional product/process properties of importance, namely the mean size of the crystals obtained, the fraction of solute recovered by the process and the process' productivity. We have applied the concept to a model system (L-glutamic acid crystallized from water as a solvent) and have shown that the results obtained are in good agreement with earlier studies conducted without breakage ${ }^{28,31}$, as well as a recent study conducted with milling ${ }^{38}$.

The parametric analysis of the single stage MSMPRC process presented here generates deep insight into the process behavior in terms of the features considered, i.e., polymorph generated, mean size, recovery and productivity. We believe that such an in-depth process understanding represents a useful stepping stone towards possible future developments for the design of continuous crystallization processes producing polymorphic materials. For in- 
stance, from our analysis, it is but a small step towards generating process alternatives that are (Pareto-)optimal in the characteristics considered; for example through the combination of the attainable region methodology introduced earlier ${ }^{33,62,65}$ and appropriate multiobjective optimization algorithms. When doing so, potential gains available when moving towards multi-stage crystallization processes should be considered as well, while, of course, paying attention to the increased complexity that such processes carry with them. 


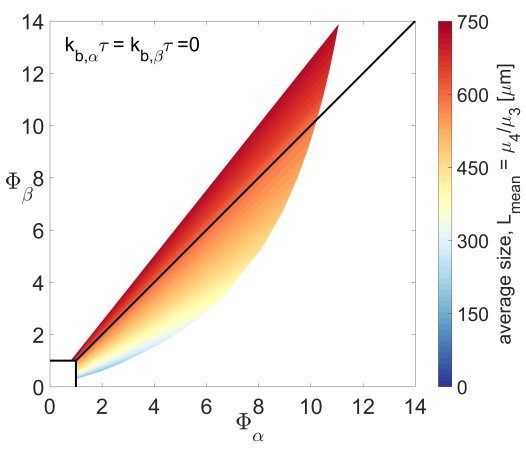

(a)

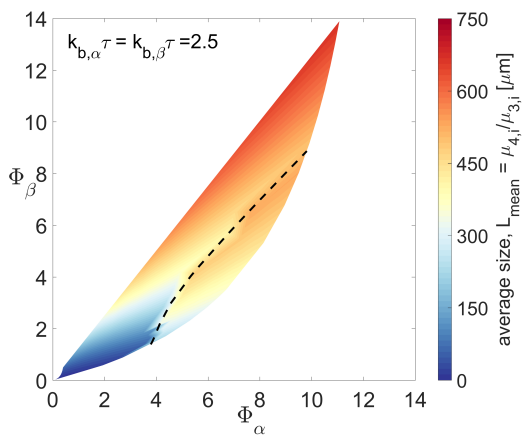

(d)

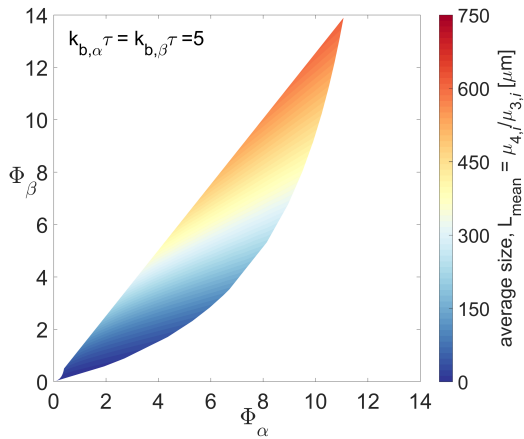

(g)

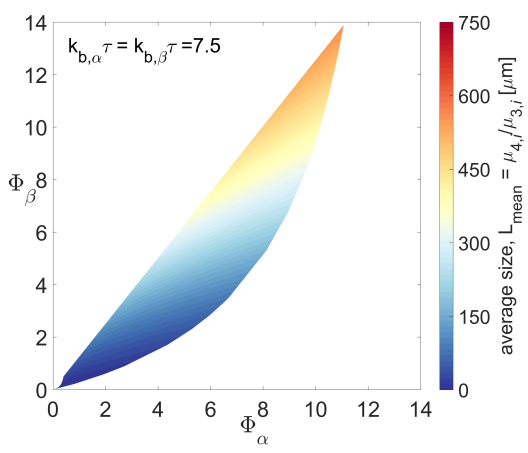

(j)

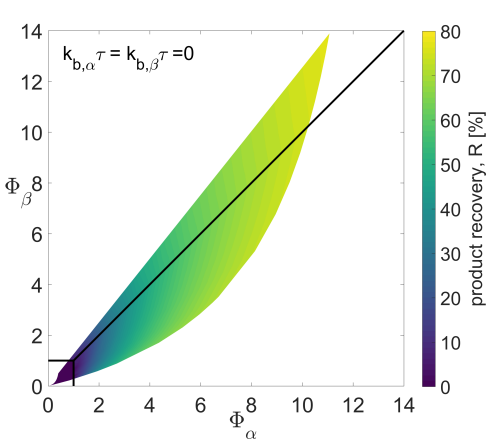

(b)

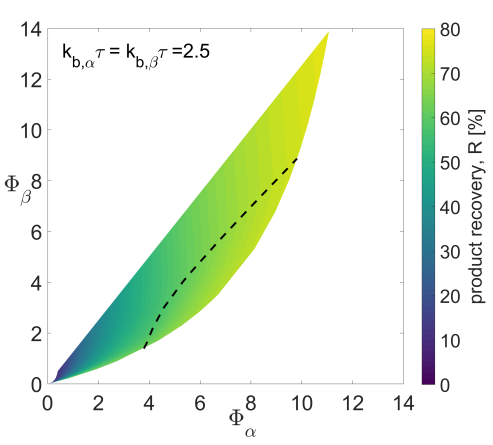

(e)

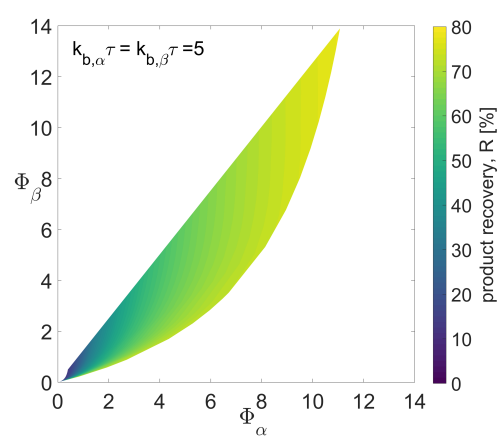

(h)

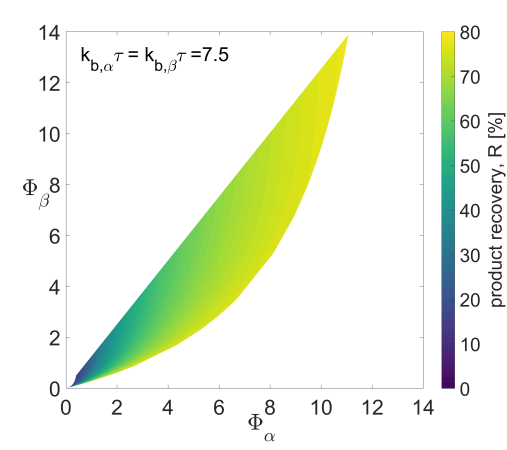

(k)

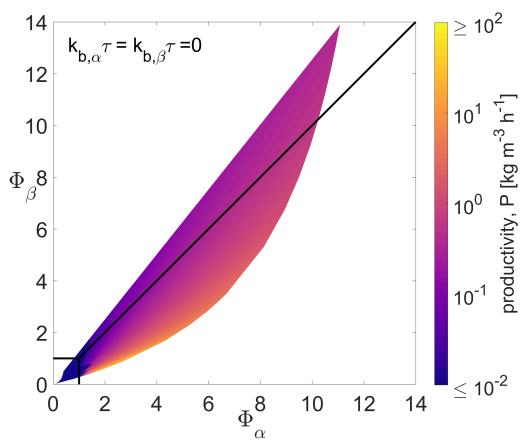

(c)

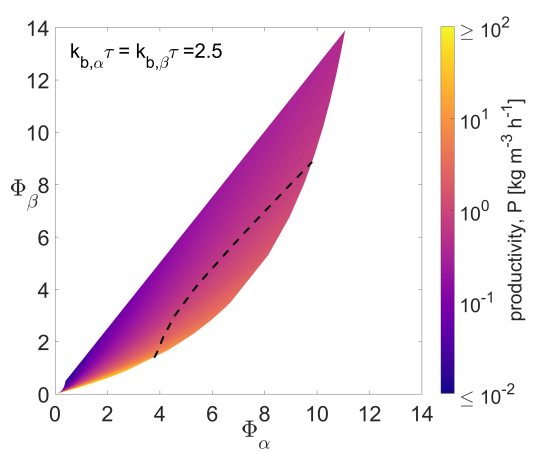

(f)

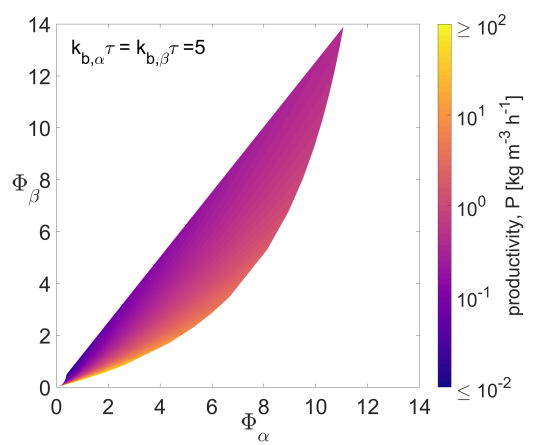

(i)

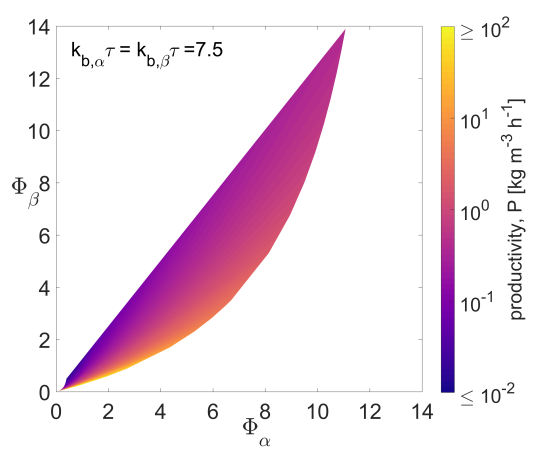

(l)

Figure 8: Volume-weighted mean size (left panels), fraction of solute recovered (middle panels), and process productivity (right panels) for every feasible operating point in the $\left(\Phi_{\alpha}, \Phi_{\beta}\right)$ plane for the model system LGA crystallized from water . The breakage intensity chosen to generate the figures increases from the top row to the bottom row (as indicated in each panel). The black lines represent the staglility boundaries (only present in the first two rows; in the third and fourth row these boundaries would lie outside the region of feasible operating points). Note that the colorscale for productivity ${ }^{64}$ is logarithmic. 
${ }_{487}$ Notation

\section{Roman Letters}

$f_{i} \quad$ number density of polymorph $i$ (per volume of suspension) $\quad\left[\mathrm{m}^{-4}\right]$

$f_{\text {seed }, i}$ number density of the seed crystals for polymorph $i$ (per vol- $\left[\mathrm{m}^{-4}\right]$ ume of suspension)

$L \quad$ particle length

$[\mathrm{m}]$

$x_{i} \quad$ dimensionless crystal size of polymorph $i$

$G_{i} \quad$ growth rate of polymorph $i$

$[-]$

$k_{g, i} \quad$ rate constant in growth rate expression for polymorph $i$

$g_{i} \quad$ exponents in power law expression for growth

$J_{i} \quad$ nucleation reate of polymorph $i$

$k_{n, i} \quad$ rate constant in nucleation rate expression for polymorph $i$

$n_{i} \quad$ exponents in power law expression for nucleation

$K_{i} \quad$ breakage rate of polymorph $i$

$k_{\mathrm{b}, i} \quad$ brekage rate prefactor

$b_{i} \quad$ exponents in power law expression for breakage rate

$L_{0, i} \quad$ scaling factor for partice length

$d_{i} \quad$ daughter distribution of polymorph $i$

$q_{i} \quad$ constant in the daughter distribution

$k_{v, i} \quad$ volumetric shape factor for polymorph $i$

$t \quad$ time

$c_{0} \quad$ inlet solute concentration

$c_{\mathrm{sat}, i} \quad$ solubility of polymorph $i$

$c \quad$ solute concentration

$c_{\mathrm{ss}} \quad$ steady state concentration

$\mathrm{D}_{\mathrm{a} i} \quad$ modified Damköhler numbers of polymorph $i$ $\left[\mathrm{m} \mathrm{s}^{-1}\right]$

$\left[\mathrm{m} \mathrm{s}^{-1}\right]$

$[-]$

[\# $\left.\mathrm{m}^{-3} \mathrm{~s}^{-1}\right]$

$\left[\# \mathrm{~m}^{-2} \mathrm{~s}^{-1}\right]$

[-]

$\left[\mathrm{s}^{-1}\right]$

$\left[\mathrm{s}^{-1}\right]$

[-]

[m]

$\left[\mathrm{m}^{-1}\right]$

$[-]$

[-]

[s]

[kg m ${ }^{-3}$ water]

[kg m${ }^{-3}$ water]

[kg m${ }^{-3}$ water $]$

[kg m${ }^{-3}$ water]

$[-]$ 
$h_{i} \quad$ dimensionless number density distribution of polymorph $i \quad[-]$

y dimensionless concentration

489

\section{Greek Letters}

$\Phi_{i} \quad$ dimensionless stability group of polymorph $i$

$\lambda \quad$ size of parent particle

$\rho_{i} \quad$ crystal density of polymorph $i$

$\tau \quad$ residence time

$\xi \quad$ dimensionless time

$\gamma \quad$ parameter that quantifies the solubility difference between two

polymorphs

$\mu_{j, i} \quad j$ th moment of the number density of polymorph $i$

$\omega_{j, i} \quad j$ th moment of the dimensionless number density of poly- [-] morph $i$

\section{Abbreviations}

MSMPRC mixed suspension, mixed product removal crystallizer

LGA L-glutamic acid

PBE population balance equation

\section{Acknowledgments}

TV thanks the Royal Academy of Engineering for support through an Engineering for Development research fellowship. YL acknowledges the funding from the China Scholarship Council (CSC). 


\section{${ }_{499}$ Supporting Information Available}

The supporting information shows the derivation of the dimensionless model equations and contains details about the numerical technique used to solve the resulting integro-differential equations, as well as further simulation results where the (less important) parameters not varied in the main part of this work are varied. 


\section{References}

(1) McCrone, W. C. In Phys. Chem. Org. Solid. State.; Fox, D., Labes, M. M., Weissberger, A., Eds.; Interscience Publishers: New York, 1965; p 726.

(2) Bernstein, J. Polymorph. Mol. Cryst.; Oxford University Press Inc.: New York, 2010; p 2.

(3) Lee, A. Y.; Erdemir, D.; Myerson, A. S. Crystal Polymorphism in Chemical Process Development. Annu. Rev. Chem. Biomol. Eng. 2011, 2, 259-280.

(4) Li, Q.; Ma, Y.; Oganov, A. R.; Wang, H.; Wang, H.; Xu, Y.; Cui, T.; Mao, H. K.; Zou, G. Superhard monoclinic polymorph of carbon. Phys. Rev. Lett. 2009, 102, 1775506.

(5) Mirmehrabi, M.; Rohani, S.; Murthy, K. S.; Radatus, B. Characterization of tautomeric forms of ranitidine hydrochloride: Thermal analysis, solid-state NMR, X-ray. J. Cryst. Growth 2004, 260, 517-526.

(6) Barbarella, G.; Zambianchi, M.; Antolini, L.; Ostoja, P.; Maccagnani, P.; Bongini, A.; Marseglia, E. A.; Tedesco, E.; Gigli, G.; Cingolani, R. Solid-state conformation, molecular packing, and electrical and optical properties of processable $\beta$-methylated sexithiophenes. J. Am. Chem. Soc. 1999, 121, 8920-8926.

(7) Bučar, D. K.; Lancaster, R. W.; Bernstein, J. Disappearing Polymorphs Revisited. Angew. Chemie - Int. Ed. 2015, 54, 6972-6993.

(8) Kobayashi, Y.; Ito, S.; Itai, S.; Yamamoto, K. Physicochemical properties and bioavailability of carbamazepine polymorphs and dihydrate. Int. J. Pharm. 2000, 193, 137-146.

(9) Müller, M.; Meier, U.; Wieckhusen, D.; Beck, R.; Pfeffer-Hennig, S.; Schneeberger, R. Process development strategy to ascertain reproducible API polymorph manufacture. Cryst. Growth Des. 2006, 6, 946-954.

(10) Chemburkar, S. R.; Bauer, J.; Deming, K.; Spiwek, H.; Patel, K.; Morris, J.; Henry, R.; Spanton, S.; Dziki, W.; Porter, W.; Quick, J.; Bauer, P.; Donaubauer, J.; Narayanan, B. A.; Soldani, M.; Riley, D.; McFarland, K. Dealing with the impact of ritonavir polymorphs on the late stages of bulk drug process development. Org. Process Res. Dev. 2000, 4, 413-417.

(11) Perini, G.; Salvatori, F.; Ochsenbein, D. R.; Mazzotti, M.; Vetter, T. Filterability prediction of needle-like crystals based on particle size and shape distribution data. Sep. Purif. Technol. 2018, 211, 768-781.

(12) Miller, J. M.; Collman, B. M.; Greene, L. R.; Grant, D. J.; Blackburn, A. C. Identifying the stable polymorph early in the drug discovery-development process. Pharm. Dev. Technol. 2005, 291-297.

(13) Llinàs, A.; Goodman, J. M. Polymorph control: past, present and future. Drug Discov. Today 2008, 13, 198-210. 
(14) Hermanto, M. W.; Chiu, M. S.; Woo, X. Y.; Braatz, R. D. Robust optimal control of polymorphic transformation in batch crystallization. AIChE J. 2007, 2643-2650.

(15) Blagden, N.; de Matas, M.; Gavan, P. T.; York, P. Crystal engineering of active pharmaceutical ingredients to improve solubility and dissolution rates. Adv. Drug Deliv. Rev. 2007, 59, 617-630.

(16) Foster, J. A.; Damodaran, K. K.; Maurin, A.; Day, G. M.; Thompson, H. P.; Cameron, G. J.; Bernal, J. C.; Steed, J. W. Pharmaceutical polymorph control in a drug-mimetic supramolecular gel. Chem. Sci. 2016, 8, 78-84.

(17) Du, W.; Yin, Q.; Gong, J.; Bao, Y.; Zhang, X.; Sun, X.; Ding, S.; Xie, C.; Zhang, M.; Hao, H. Effects of solvent on polymorph formation and nucleation of prasugrel hydrochloride. Cryst. Growth Des. 2014, 14, 4519-4525.

(18) Parambil, J. V.; Poornachary, S. K.; Tan, R. B.; Heng, J. Y. Influence of solvent polarity and supersaturation on template-induced nucleation of carbamazepine crystal polymorphs. J. Cryst. Growth 2017, 469, 84-90.

(19) Zhu, L.; Wang, L. Y.; Sha, Z. L.; Wang, Y. F.; Yang, L. B.; Zhao, X. Y.; Du, W. Interplay between Thermodynamics and Kinetics on Polymorphic Appearance in the Solution Crystallization of an Enantiotropic System, Gestodene. Cryst. Growth Des. 2017, 17, 4582-4595.

(20) Beckmann, W. Seeding the desired polymorph: Background, possibilities, limitations, and case studies. Org. Process Res. Dev. 2000, 4, 372-383.

(21) Hiremath, R.; Basile, J. A.; Varney, S. W.; Swift, J. A. Controlling molecular crystal polymorphism with self-assembled monolayer templates. J. Am. Chem. Soc. 2005, 127, $18321-18327$.

(22) Agnew, L. R.; Cruickshank, D. L.; McGlone, T.; Wilson, C. C. Controlled production of the elusive metastable form II of acetaminophen (paracetamol): A fully scalable templating approach in a cooling environment. Chem. Commun. 2016, 52, 7368-7371.

(23) Kitamura, M. Strategy for control of crystallization of polymorphs. CrystEngComm 2009, 11, 949-964.

(24) Kee, N. C.; Tan, R. B.; Braatz, R. D. Selective crystallization of the metastable $\alpha$-form of L-glutamic acid using concentration feedback control. Cryst. Growth Des. 2009, 9, 3044-3051.

(25) Derdour, L.; Skliar, D. A review of the effect of multiple conformers on crystallization from solution and strategies for crystallizing slow inter-converting conformers. Chem. Eng. Sci. 2014, 106, 275-292.

(26) Simone, E.; Saleemi, A. N.; Tonnon, N.; Nagy, Z. K. Active polymorphic feedback control of crystallization processes using a combined raman and ATR-UV/Vis spectroscopy approach. Cryst. Growth Des. 2014, 14, 1839-1850. 
(27) Simone, E.; Szilagyi, B.; Nagy, Z. K. Systematic model identification and optimizationbased active polymorphic control of crystallization processes. Chem. Eng. Sci. 2017, $174,374-386$.

(28) Lai, T. T. C.; Ferguson, S.; Palmer, L.; Trout, B. L.; Myerson, A. S. Continuous crystallization and polymorph dynamics in the 1 -glutamic acid system. Org. Process Res. Dev. 2014, 18, 1382-1390.

(29) Lai, T.-T. C.; Cornevin, J.; Ferguson, S.; Li, N.; Trout, B. L.; Myerson, A. S. Control of Polymorphism in Continuous Crystallization via Mixed Suspension Mixed Product Removal Systems Cascade Design. Cryst. Growth Des. 2015, 15, 3374-3382.

(30) Agnew, L. R.; McGlone, T.; Wheatcroft, H. P.; Robertson, A.; Parsons, A. R.; Wilson, C. C. Continuous Crystallization of Paracetamol (Acetaminophen) Form II: Selective Access to a Metastable Solid Form. Cryst. Growth Des. 2017, 17, 2418-2427.

(31) Farmer, T. C.; Carpenter, C. L.; Doherty, M. F. Polymorph selection by continuous crystallization. AIChE J. 2016, 62, 3505-3514.

(32) Farmer, T. C.; Schiebel, S. K.; Chmelka, B. F.; Doherty, M. F. Polymorph Selection by Continuous Precipitation. Cryst. Growth Des. 2018, 18, 4306-4319.

(33) Vetter, T.; Burcham, C. L.; Doherty, M. F. Separation of conglomerate forming enantiomers using a novel continuous preferential crystallization process. AIChE J. 2015, $61,2810-2823$.

(34) Köllges, T.; Vetter, T. Model-based analysis of continuous crystallization/reaction processes separating conglomerate forming enantiomers. Cryst. Growth Des. 2017, 17, $233-247$.

(35) Yang, Y.; Song, L.; Zhang, Y.; Nagy, Z. Application of wet milling-based automated direct nucleation control in continuous cooling crystallization processes. Ind. Eng. Chem. Res. 2016, 55, 4987-4996.

(36) Acevedo, D.; Kamaraju, V. K.; Glennon, B.; Nagy, Z. K. Modeling and Characterization of an in Situ Wet Mill Operation. Org. Process Res. Dev. 2017, 21, 1069-1079.

(37) Yang, Y.; Song, L.; Gao, T.; Nagy, Z. Integrated upstream and downstream application of wet milling with continuous mixed suspension mixed product removal crystallization. Cryst. Growth Des. 2015, 15, 5879-5885.

(38) Köllges, T.; Vetter, T. Polymorph selection and process intensification in a continuous crystallization-milling process: A case study on L-glutamic acid crystallized from water. Org. Process Res. Dev. doi: 10.1021/acs.oprd.8b00420.

(39) Randolph, A. D.; Larson, M. A. Theory of Particulate Processes: : Analysis and Techniques of Continuous Crystallization, 2nd ed.; Academic Press Inc: San Diego, California, 1988; pp 11-13. 
(40) Ramkrishna, D. Population Balance: Theory and Applications to Particulate Systems in Engineering; Academic Press: San Diego, 2000.

(41) Köllges, T.; Vetter, T. Design and Performance Assessment of Continuous Crystallization Processes Resolving Racemic Conglomerates. Cryst. Growth Des. 2018, 18, 1686-1696.

(42) Iggland, M.; Mazzotti, M. A population balance model for chiral resolution via Viedma ripening. Cryst. Growth Des. 2011, 11, 4611-4622.

(43) Salvatori, F.; Mazzotti, M. Manipulation of particle morphology by crystallization, milling, and heating cycles: experimental characterization. Ind. Eng. Chem. Res. 2018, $5 \%, 15522-15533$.

(44) Qamar, S.; Elsner, M. P.; Angelov, I. A.; Warnecke, G.; Seidel-Morgenstern, A. A comparative study of high resolution schemes for solving population balances in crystallization. Comput. Chem. Eng. 2006, 30, 1119-1131.

(45) Qamar, S.; Warnecke, G.; Elsner, M. P. On the solution of population balances for nucleation, growth, aggregation and breakage processes. Chem. Eng. Sci. 2009, 64, 2088-2095.

(46) Kumar, R.; Kumar, J. Numerical simulation and convergence analysis of a finite volume scheme for solving general breakage population balance equations. Appl. Math. Comput. 2013, 219, 5140-5151.

(47) Ohara, M.; Reid, R. C. Modelling Crystal Growth Rates from Solutions; Prentice-Hall, Inc.: Englewood Cliffs, N. J., 1973.

(48) Tseng, Y. T.; Ward, J. D. Critical seed loading from nucleation kinetics. AIChE J. 2014, 60, 1645-1653.

(49) Austin, L.; Shoji, K.; Bhatia, V.; Jindal, V.; Savage, K.; Klimpel, R. Some Results on the Description of Size Reduction as a Rate Process in Various Mills. Ind. Eng. Chem. Process Des. Dev. 1976, 15, 187-196.

(50) Luciani, C. V.; Conder, E. W.; Seibert, K. D. Modeling-aided scale-up of high-shear rotor-stator wet milling for pharmaceutical applications. Org. Process Res. Dev. 2015, $19,582-589$.

(51) Kitamura, M. Polymorphism in the crystallization of L-glutamic acid. J. Cryst. Growth 1989, 96, 541-546.

(52) Manzurola, E.; Apelblat, A. Solubilities of L-glutamic acid, 3-nitrobenzoic acid, p-toluic acid, calcium-L-lactate, calcium gluconate, magnesium-DL-aspartate, and magnesiumL-lactate in water. J. Chem. Thermodyn. 2002, 34, 1127-1136.

(53) Schöll, J.; Bonalumi, D.; Vicum, L.; Mazzotti, M.; Müller, M. In situ monitoring and modeling of the solvent-mediated polymorphic transformation of L-glutamic acid. Cryst. Growth Des. 2006, 6, 881-891. 
(54) Lindenberg, C.; Schöll, J.; Vicum, L.; Mazzotti, M.; Brozio, J. L-glutamic acid precipitation: agglomeration effects. Cryst. Growth Des. 2008, 8, 224-237.

(55) Cornel, J.; Lindenberg, C.; Mazzotti, M. Experimental characterization and population balance modeling of the polymorph transformation of L-glutamic acid. Cryst. Growth Des. 2008, 9, 243-252.

(56) Hermanto, M. W.; Chiu, M. S.; Braatz, R. D. Nonlinear Model Predictive Control for the Polymorphic Transformation of L-Glutamic Acid Crystals. AIChE J. 2009, 55, 2631-2645.

(57) Mo, Y.; Dang, L.; Wei, H. Solubility of alpha-form and beta-form of l-glutamic acid in different aqueous solvent mixtures. Fluid Phase Equilib. 2011, 300, 105-109.

(58) Ochsenbein, D.; Schorsch, S.; Vetter, T.; Mazzotti, M.; Morari, M. Growth Rate Estimation of $\beta$ L-Glutamic Acid from Online Measurements of Multidimensional Particle Size Distributions and Concentration. Ind. Eng. Chem. Res. 2014, 53, 9136-9148.

(59) Lehmann, M. S.; Nunes, A. C. A short hydrogen bond between near identical carboxyl groups in the $\alpha$-modification of L-glutamic acid. Acta Crystallogr. Sect. B Struct. Crystallogr. Cryst. Chem. 1980, 36, 1621-1625.

(60) Hirokawa, S. A new modification of L-glutamic acid and its crystal structure. Acta Crystallogr. 1955, 8, 637-641.

(61) Vetter, T.; Burcham, C. L.; Doherty, M. F. Designing robust crystallization processes in the presence of parameter uncertainty using attainable regions. Ind. Eng. Chem. Res. 2015, 54, 10350-10363.

(62) Wang, J.; Lakerveld, R. Continuous Membrane-Assisted Crystallization To Increase the Attainable Product Quality of Pharmaceuticals and Design Space for Operation. Ind. Eng. Chem. Res. 2017, 56, 5705-5714.

(63) Wong, S. Y.; Tatusko, A. P.; Trout, B. L.; Myerson, A. S. Development of continuous crystallization processes using a single-stage mixed-suspension, mixed product removal crystallizer with recycle. Cryst. Growth Des. 2012, 12, 5701-5707.

(64) Hunter, J. D. Matplotlib: A 2D graphics environment. Computing In Science 8$\}$ Engineering 2007, 9, 90-95.

(65) Vetter, T.; Burcham, C. L.; Doherty, M. F. Regions of attainable particle sizes in continuous and batch crystallization processes. Chem. Eng. Sci. 2014, 106, 167-180. 
${ }_{681}$ For Table of Contents Use Only

Title: Polymorph selection by continuous crystallization in the presence of wet milling

Author: Yang Li, Simon O’Shea, Qiuxiang Yin, Thomas Vetter

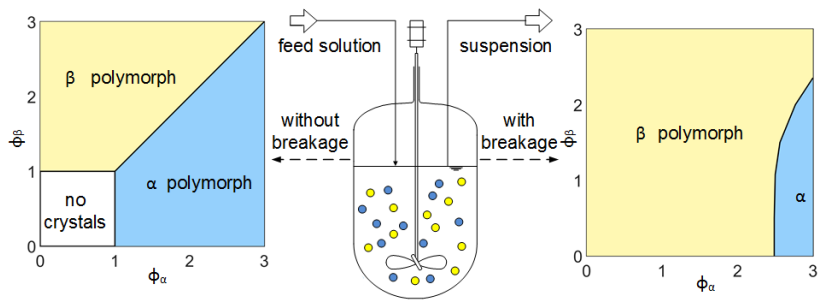

Synopsis: We explore the ability of combined continuous crystallization-milling processes (based on a single stage mixed suspension mixed product removal crystallizer) to reliably produce a desired polymorph and investigate the ranges the combinations of mean sizes, fraction of solute recovered and process productivity that are feasible in such processes. 